\title{
Nascent Polypeptide Associated Complex-alpha and Signal Recognition Particle are required for cardiac development and remodeling
}

\author{
Analyne M. Schroeder ${ }^{1, \star}$, Georg Vogler ${ }^{1}$, Alexandre R. Colas ${ }^{1}$, Rolf Bodmer ${ }^{1, \star}$ \\ ${ }^{1}$ Development, Aging and Regeneration Program, Sanford Burnham Prebys Medical Discovery \\ Institute, La Jolla, CA, USA
}

* Corresponding authors:

\begin{abstract}
Congenital Heart Disease (CHD) is driven by a strong genetic predisposition, yet only a small subset of patients $(\sim 20 \%)$ are diagnosed with a precise genetic cause. Therefore, expanding the pool of genes associated with CHD and establishing the functional relationships between genes can assemble a more comprehensive genetic network to better understand cardiac development and pathogenesis. In our studies, we identified protein biogenesis cofactors Nascent polypeptide Associated Complex (NAC) and Signal Recognition Particle (SRP) that bind disparate subsets of emerging nascent polypeptides at the ribosome exit site to direct polypeptide fates, as novel regulators of cell differentiation and cardiac morphogenesis. Knockdown (KD) of the alpha- (Naco) or beta- subunit (bicaudal, bic) of NAC in the developing Drosophila heart led to disruption of cardiac remodeling during pupal stages resulting in an adult fly with no heart. Heart loss was rescued by combined KD of Nac $\alpha$ with the Hox gene Abd-B. Consistent with a central role for this interaction in the regulation of cardiogenesis, KD of Nac $\alpha$ in Cardiac Progenitors derived from human iPSCs impaired cardiac differentiation while co-KD with mammalian Hox genes HOXC12 and HOXD12 rescued this phenotype. The effect of Nac $\alpha$ KD on the fly heart was temporally regulated, in that KD in embryo or in pupae caused only a partial loss of the heart, whereas KD during both stages led to heart loss similar to continuous KD throughout life. This suggests that Nac $\alpha$ KD already in the embryo may reprogram cells leading to aberrant cardiac remodeling during pupal stages. Lastly, KD of several SRP subunits individually in the fly heart produced a range of cardiac phenotypes that targeted specific segments and cell types, indicating spatially regulated activities of SRP components in the heart. Together, these data suggest that despite NAC and SRP ubiquitous presence, they displayed
\end{abstract}


bioRxiv preprint doi: https://doi.org/10.1101/2022.01.24.477419; this version posted January 24, 2022. The copyright holder for this preprint (which was not certified by peer review) is the author/funder, who has granted bioRxiv a license to display the preprint in perpetuity. It is made available under aCC-BY-NC-ND 4.0 International license.

spatially and temporally fine-tuned activities for proper cardiac morphogenesis. Nac $\alpha$ 's interaction with cardiac-specific Hox gene functions builds upon the novel role of this pathway and expands our understanding of the complex genetic networks involved in cardiac development and pathogenesis. 


\section{INTRODUCTION}

Congenital Heart Disease (CHD) is characterized by structural malformations of the heart present at birth caused by deviations from the normal course of cardiogenesis [1]. Genetics is a critical driver of CHD [2, 3]. Chromosomal anomalies as well as variants in genes involved in heart development have been identified in CHD patients [4]. These genetic features and disease presentations are heritable and cluster in families [5, 6]. Identifying the genes associated with disease helps piece together genetic networks that could uncover mechanisms underlying pathogenesis. Approximately 400 genes have been implicated in $\mathrm{CHD}$ [2], some that cluster within defined pathways, which permits a genetic diagnosis for approximately $20 \%$ of CHD patients. However, this leaves the vast majority of CHD cases with unknown genetic origins [3]. Therefore, expansion of the genetic data pertinent to CHD, such as functional analysis of genes with variants of uncertain significance (VUS), would advance our understanding of the disease and may offer, in the future, a diagnosis and targeted treatment for CHD patients. A better understanding of additional genetic risk factors and patient-specific combinations of such factors is aided by identification of candidate disease genes through patient-specific genomics, such as whole genome sequencing (WGS), followed by their evaluation in cardiac developmental platforms and assays from various genetic model systems [7, 8]. These validation efforts can accelerate candidate gene identification and focus on new potentially pathogenic genes, including genes located within larger genomic anomalies such as de novo Copy Number Variants [9].

Previous data suggest that the human gene Nascent polypeptide Associated Complexalpha (NACA) is a candidate CHD gene that could provide novel insights into biological pathways in cardiac morphogenesis and pathogenesis. Using a GWAS approach, NACA was located within a genomic locus associated with increased myocardial mass [10], while Whole Exome Sequencing in families with Tetralogy of Fallot identified a single nucleotide polymorphism within NACA [11]. NACA is a highly conserved alpha subunit of a heterodimeric complex called Nascent polypeptide Associated Complex (NAC). Along with its heterodimeric partner, NAC-beta (NACB/BTF3), NAC is one of several chaperones found near the ribosome exit tunnel that bind to select emerging nascent polypeptides [12]. NAC-ribosome complexes facilitate transport of nascent polypeptides to the mitochondria as has been demonstrated in yeast [13-15]. At the ribosome exit site, NAC gates the activity of other nascent polypeptide chaperones. For example, NAC enhances the fidelity of Signal Recognition Particle (SRP) binding to only those nascent polypeptides destined for import to the Endoplasmic Reticulum (ER) [16-18]. Depletion of NAC leads to promiscuous binding of SRP onto nascent polypeptides, drawing mistargeted ribosome- 
nascent polypeptide complexes to the ER for aberrant insertion into the membrane or secretion. NACA's function as part of NAC therefore regulates the localization and posttranslational quality control of proteins in the cell.

In Drosophila, NAC plays an important role in translational regulation critical for embryonic development [19, 20]. Fly homologs of NAC subunits, Nac $\alpha$ and bicaudal (bic), were shown to repress protein translation of the posterior patterning gene Oskar (Osk) in anterior regions of the embryo, which was despite association of osk mRNA with polysomes, usually indicative of active translation. Restricting Osk protein translation and accumulation to the posterior pole of the embryo is critically required for patterning the posterior body plan [21]. Depletion of either Nac $\alpha$ or bic, expands Osk protein localization anteriorly resulting in a bicaudal phenotype, where the embryo develops with mirror-image duplication of the posterior axis [19, 22]. These results suggest that within the developing embryo syncytium, NAC appears to regulate the expression of select proteins for proper spatial distribution. NAC could have a similar role in the timing and spatial targeting of translation within specific tissues.

Nac $\alpha$ has been demonstrated to be critical for development of several tissues using various model organisms. In mouse, the Nac $\alpha$ subunit can function as a transcriptional coactivator regulating bone development [23-25] and hematopoiesis in zebrafish [26]. In vertebrates, a skeletal muscle- and heart-specific variant of Nac $\alpha$ has been associated with myofibril organization in zebrafish [27] and muscle and bone differentiation in mouse [28-32]. Recent studies in the fly showed that Nac $\alpha$ knockdown (KD) specifically in the heart led to a 'no adult heart' phenotype [10], suggesting that Nac $\alpha$ could play a role in sarcomeric biogenesis, but its exact role in cardiac development had been unclear.

Here, we provide evidence for a cardiac developmental role for $N A C$ and $S R P$ in Drosophila and NACA in human Multipotent Cardiac Progenitors (MCPs). In flies, cardiac KD of $N A C \alpha$ (mentioned above) and bic throughout development led to complete loss of the heart. We demonstrate that this phenotype is dependent on the timing of $N A C \alpha \mathrm{KD}$, which requires $\mathrm{KD}$ during both embryonic heart development and pupal cardiac remodeling from larval to adult heart for a complete loss of the heart. KD in embryos only led to loss of the terminal chamber of the adult heart, while retaining heart structures in the anterior segments. KD of Nac $\alpha$ only during pupal stages did not affect adult heart structure. This suggests that NAC $\alpha$ KD primes cardiac cells 
already in the embryo for aberrant responses to morphogenic cues during later developmental stages. Persistent Nac $\alpha$ KD during pupal stages remained required for complete loss of the adult heart. Consistent with this idea, $N A C \alpha$ KD throughout cardiac development induced ectopic expression of the posterior patterning hox gene $A b d o m i n a l-B(A b d-B)$ into anterior regions of the remodeling heart during pupation. Concurrent KD of $N A C \alpha$ and $A b d-B$ partially rescued the heart. This interaction between Nac $\alpha$ and Hox gene was recapitulated in MCPs, whereby NACA KD led to deviations in progenitor cell differentiation away from cardiomyocytes and toward fibroblast cell fates, which was reversed with combined KD of NACA and Hox genes HOXC12 an HOXD12. Because NAC associates and influences the activity of SRP, we tested the effects of individual SRP subunit KD on the fly heart. Interestingly, KD of individual SRP subunits produced cardiac phenotypes that were distinctly different from those of $N A C \alpha$, affecting other heart regions and targeting specific cell types. These results suggest specific roles for ubiquitously expressed protein biogenesis factors NAC and SRP in heart morphogenesis, in part through alterations in the expression of body patterning proteins. Translational regulation adds to our growing knowledge of biological pathways that may specifically contribute to cardiac pathogenesis leading to CHD.

\section{RESULTS}

\section{Knockdown of $\mathrm{Nac} \alpha$ and bicaudal in the heart throughout development results in complete absence of the adult heart.}

The Nascent Polypeptide Complex (NAC) is a heterodimeric complex made of an alpha (NACA/Nac $\alpha$ ) and beta (NAC $\beta / B T F 3 /$ bicaudal) subunit that bind ribosomes to influence translation, protein folding, and transport of select nascent polypeptide chains. We investigated the role of the NAC complex, focusing on the alpha subunit, in the development and function of the Drosophila heart. Consistent with previously published data [10], knockdown (KD) of Nac $\alpha$ by RNAi using the Hand4.2-GAL4 driver [33] that is active in the heart tube and surrounding pericardial cells throughout development, led to a 'no-heart' phenotype in adults when stained with phalloidin and the heart specific collagen, pericardin (Figure 1A,B). Similarly, KD of the $\beta$ subunit, bicaudal, using Hand4.2-GAL4 also led to a 'no-heart' phenotype (Figure 1C). Despite Nac $\alpha$, or bic KD throughout heart development and the absence of hearts in adults, the posterior 
aorta and heart tube was still present in controls, Nac $\alpha$ KD, and bic KD during early pupae as captured by in vivo imaging of fluorescently labeled hearts (Figure 1D-F). We filmed and followed cardiac remodeling through pupation (Supplemental Video 1-3, Supplemental Figure 1A). Within the first 24 hours of pupation, hearts were detected in both controls and Nac $\alpha$ KD flies. Normally, the heart undergoes remodeling through trans-differentiation of the larval aorta into the adult heart tube (Figure 3A). However, after 24-28 hours of puparium formation (APF) the heart of cardiac Nac $\alpha$ KD flies began to disappear as cardiac remodeling progressed (Supplemental Figure 1A). The space at the dorsal midline usually taken up by the heart, is now filled in with fat cells, indicating that the heart undergoes complete histolysis during remodeling. A similar course of events is observed when bicaudal is knocked down in the heart (Supplemental Video 3; Supplemental Figure 1A). We also stained Nac $\alpha$ KD hearts with phalloidin just prior to cardiac remodeling (about 26-28 AFP), where heart tubes were present, but were narrower with disorganized actin filament arrangement (Supplemental Figure 1C).

Using cardiomyocyte-specific drivers conferring weaker expression than Hand4.2-GAL4 to reduce Nac $\alpha$ levels, i.e. tinHE-GAL4 and $\operatorname{tin} C \Delta 4-G A L 4$, did not produce severe heart loss but altered several parameters of heart function and structure, as measured by SOHA (see methods; Figure 2A,B). Diastolic diameters were decreased using both drivers (Figure 2C) without a change in systolic diameter (Figure 2D), and consequently reducing contractility, as measured by diminished fractional shortening, FS (Figure 2E). No changes were detected in heart period (Figure 2F) and diastolic intervals (Figure 2G). KD of Nac $\alpha$ with tinC $\triangle 4-G A L 4$ caused a slight reduction in systolic interval, which is the duration of active heart contraction and relaxation (Figure 2H). Phalloidin staining of the adult hearts with reduced Nac $\alpha$ expression showed gaps between the circumferential myofibrils and sarcomeric disorganization compared to controls, consistent with reduced contractility (Figure 2I). These results suggest that at certain expression thresholds where heart development is not completely diverted, Nac $\alpha$ may play a role in functional and structural aspects of the heart as well. Because Hand4.2-GAL4 drives expression in both cardiac cells and the pericardial nephrocytes, we tested the effect of Nac $\alpha$ KD in pericardial cells only using Dot-GAL4 [34] to assess their contribution to the overall heart phenotype (Figure 2 BH). KD in the pericardial cells did not produce significant changes in heart function, except for a slight increase in systolic diameter but no change in contractility (Figure 2D,E). The organization of the circumferential myofibrils as stained by phalloidin was unaltered compared to control 
(Figure 2I), suggesting that KD of Nac $\alpha$ in pericardial cells contribute minimally to the overall cardiac phenotype.

\section{Nac $\alpha$ genetically interacts with the Hox gene $A b d-B$ in heart development.}

We sought to better understand the mechanisms driving complete histolysis of the heart tube during metamorphosis. It is well established that during normal cardiac remodeling the posterior most segment of the larval heart (abdominal segments 6-7) histolyzes and is no longer present in adult hearts [35]. The posterior larval heart region destined for histolysis expresses the Hox segmentation gene Abdominal-B (Abd-B) [36-38]. Abdominal segment 5 in the larvae that expresses the hox segmentation gene abdominal-A (abd-A) remodels to become the adult terminal chamber, while the larval aorta (abdominal segment 1-4) that expresses Hox gene Ultrabithorax (Ubx) remodels to become the adult heart proper (Figure 3A). We postulated that KD of Nac $\alpha$ throughout the heart could result in the misexpression of $A b d-B$ leading to complete histolysis of the heart. First, we examined whether overexpression of $A b d-B$ throughout the heart would result in similar phenotypes as with $\operatorname{Nac} \alpha$ KD. Previously published data overexpressing $A b d-B$ using an early pan-mesodermal driver (twist-GAL4) led to severely diminished embryonic muscle and heart development [39]. Using the cardiac-specific driver Hand4.2-GAL4 to overexpress $A b d-B$, the heart was completely absent in adults (Figure 3B, right), however a heart tube was present in larvae and in early pupae (24 hours APF, Figure 3D), similar to control (Figure 3C) Nac $\alpha$-RNAi (Figure 3E) and bic-RNAi phenotypes (Figure 1). These results suggest that $A b d-B$ expression and activity are highly temporally controlled, and drive cardiomyocyte histolysis only during cardiac remodeling at pupal stages.

We then examined whether KD of $N a c \alpha$ leads to ectopic expression of Abd-B protein in the early pupal heart just prior to remodeling (APF 26-28). In controls, Abd-B protein is undetected in the anterior regions of the early pupal heart tube (Figure $3 \mathrm{C}$ ), but detected in the posterior segments of the embryo. Overexpression of $A b d-B$ using Hand4.2-GAL4 led to increased Abd-B protein accumulation throughout the early pupal heart tube and pericardial cells, which was restricted to the nuclei (Figure 3D). When Nac $\alpha$ was knocked down in the heart, we detected Abd-B protein expression throughout the larval/early pupal heart tube, within the myocardial nuclei as well as in the cytoplasm (Figure 3E). In orthogonal sections, there is ectopic Abd-B staining centrally, possibly within the lumen of the heart, either secreted from cardiomyocytes or bound to 
circulating hemocytes. These data suggest that KD of Nac $\alpha$ in the heart led to ectopic expression of Abd-B within the cell, as well as, anteriorly throughout the heart tube. Because overexpression of Abd-B using Hand4.2-GAL4 led to heart histolysis during remodeling, we speculate that ectopic $\mathrm{Adb}-\mathrm{B}$ expression induced by $\mathrm{Nac} \alpha \mathrm{KD}$ is likely leading to the observed histolysis of the entire heart during remodeling.

Because Nac $\alpha$ KD led to ectopic expression of Abd-B, we tested whether the no-heart phenotype produced by Nac $\alpha$ KD could be rescued by concurrent KD of $A b d-B$. KD of $A b d-B$ alone led to largely intact hearts except for posterior ends that were more dilated and prominent compared to controls suggesting incomplete histolysis during cardiac remodeling (Supplemental Figure 2). KD of both $N a c \alpha$ and $A b d-B$ using the Hand4.2-GAL4 driver led to a remarkable rescue of the adult heart, restoring formation of most of the circumferential myofibrils in the anterior regions (Figure 3F,G). These hearts had reduced diastolic diameters, without change in systolic diameter that significantly reduced FS (Figure $3 \mathbf{H}$ ). This rescue was not due to titration of GAL4 protein by competing UAS sites, since a no-heart phenotype was still produced when Nac $\alpha-R N A i$ was combined with another UAS that carries a UAS-Val10-GFP construct (Figure 3I). These rescue data suggest a genetic interaction between $N a c \alpha$ and $A b d-B$. On the other hand, coexpression of $N a c \alpha-R N A i$ with an inhibitor of apoptosis (Death-associated inhibitor of apoptosis 1, DIAP1) did not rescue the heart (Supplemental Figure 3), suggesting that the Nac $\alpha$-RNAi mediated heart loss cannot simply be prevented by inhibition of canonical cell-death pathways.

We also tested whether $A b d-B$ KD could rescue the moderate cardiac function defects produced by less robust KD of Nac $\alpha$, using the tinHE-GAL4 driver, that only drives expression during embryonic development. Nac $\alpha$ KD with tinHE-GAL4 caused cardiac constriction but no effects on heart period or intervals (Supplemental Figure 4A,B). KD of $A b d-B$ using tinHE-GAL4 did not produce significant changes in heart function compared to controls, except for a prolonged systolic interval. Combining Nac $\alpha-R N A i$ with $A b d-B-R N A i$ reversed the constricted diastolic and systolic diameter phenotype of Nac $\alpha$-RNAi;UAS-Stinger::GFP, such that the phenotype mirrored controls or $A b d-B-R N A i ; U A S-S t i n g e r ~ K D$. An improvement in circumferential myofibrillar organization is also evident with co-KD of $N a c \alpha$ and $A b d-B$ (Supplemental Figure 4C). These data suggest that $A b d-B$ and $N a c \alpha$ co-KD cannot only restore heart formation with the strong Hand4.2 driver, but also normal heart function and myofibrillar organization with the weaker tinHE- 
GAL4 driver. This means that functional maturation requires $N a c \alpha$ to restrict $A b d-B$ function to the posterior of the heart.

\section{Nac $\alpha$ is required in the embryo to pre-program cardioblasts for appropriate cardiac remodeling.}

Prior to pupal cardiac remodeling, a larval heart was still present despite Naca KD using the strong cardiac driver Hand4.2-GAL4 (Figure 1E and 3E). However, these larval heart tubes were thinner and the cytoskeletal structures less prominent than controls (Supplemental Figure $1 \mathrm{~B}$ and Figure $3 \mathrm{E}$ ), reminiscent to the constricted adult heart phenotypes with the weaker driver tinHE-Gal4 (Supplemental Figure 4). These observations suggest that Naca may have earlier developmental functions in addition to a role in metamorphosis. We therefore wanted to temporally dissect Naca's function in the heart by knocking down its expression during different developmental stages. We generated a Hand4.2-GAL4 driver line that included two copies of a temperature-sensitive allele of GAL80 driven by a ubiquitous promoter (tubulin-GAL80 ${ }^{\text {ts }}$ ), which we termed HTT [9]. At the permissive temperature $\left(18^{\circ} \mathrm{C}\right)$, the GAL80 transcriptional repressor prevents GAL4 activation of UAS sites thereby inhibiting transcription of downstream constructs [40]. This temperature-sensitive form of GAL80 protein is unstable at higher temperatures (28$29^{\circ} \mathrm{C}$ ), thus permitting GAL4 activity at higher ambient temperatures.

Maintaining HTT flies crossed to Naca-RNAi or controls at $18^{\circ} \mathrm{C}$ throughout development resulted in normal heart structure (Figure 4A) and produced no differences in diastolic diameter, systolic diameter or fractional shortening compared to controls (Figure 4I), effectively demonstrating GAL80's ability to suppress Nac $\alpha-R N A i$ transcription at $18^{\circ} \mathrm{C}$ temperatures. Constant exposure to $28^{\circ} \mathrm{C}$ throughout development phenocopies the absence of the heart in adults produced by Hand4.2-GAL4 driver (Figure 4B). KD of Naca in adults only for one week by exposure to high temperatures also led to largely normal heart structure and function compared

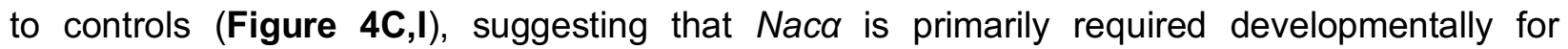
establishing a normal heart in adults, rather than maintaining its function or structure with age. Remarkably, although lifelong cardiac Naca KD using Hand4.2-GAL4 led to histolysis of the heart during metamorphosis, KD of Naca only during pupation did not produce gross heart defects (Figure 4D). The diastolic and systolic diameters were slightly increased, which caused some reduction in fractional shortening (Figure 4I). Even when we induced Naca KD earlier, starting at mid-larval stages through metamorphosis until eclosion ( $\sim 5$ days), a period with substantial 
developmental growth requiring high levels of protein translation, heart structure was unaffected (Figure 4E). This lack of phenotype is remarkable, as disruption of NAC is associated with proteostasis and ER stress, which often leads to cell death [41-43]. These results suggest that $\mathrm{KD}$ of $\mathrm{Nac \alpha}$ in the developing heart, even for relatively long durations does not unequivocally lead to cell death. When Naca was knocked down during embryonic stages only (egg-lay up to 24 hours) and subsequently reared at $18^{\circ} \mathrm{C}$ until dissection to prevent $N a c \alpha \mathrm{KD}$ at later stages, most adult hearts remained intact, but considerably constricted with smaller diastolic and systolic diameters (Figure 4F,I). Interestingly, the posterior terminal heart chamber was absent in most cases (Figure 4F), which suggests that the no-heart phenotype observed with continuous KD likely arises from developmental defects already in the embryo. Extending exposure to higher temperature during embryonic stages until 48 hours after egg lay, produced similar phenotypes compared to $24 \mathrm{hr}$ exposure, with the presence of a heart tube but without a terminal chamber (Figure 4G). Remarkably, when flies were exposed to higher temperatures during both embryonic stages (24 hours) and pupal stages (three days), with a return to $18^{\circ} \mathrm{C}$ during larval development, an almost complete 'no-heart' phenotype was reproduced in most cases (Figure $4 \mathbf{H}$ ). This suggests that Naca KD only during combined embryonic and pupal stages produces a nearly complete loss of heart structures, but at either stage alone was insufficient for such a severe phenotype.

These results suggest that Naca's role in driving heart morphogenesis has a temporal component, perhaps regulating several different processes during cardiac development. Furthermore, the observation that Naca KD during embryonic as well as pupal stages is needed to induce histolysis of nearly the entire heart tube during pupal cardiac remodeling suggests that Naca plays an essential role in the embryonic heart development by programing cardiac cell fate. This embryonic requirement seems to be partially compensated for by Naca function during heart remodeling. However, the additional reduction of pupal Naca exacerbates the lack of embryonic cardioblast programming, together leading to a failure of the larval heart to respond to remodeling cues during metamorphosis, thus causing histolysis.

\section{Nac $\alpha$ alters cell-fate in human Multipotent Cardiac Progenitors and is modulated by Hox genes.}

Since our results suggest that Nac $\alpha$ plays a role in establishing cell identity and cell-fate of cardiac cells in Drosophila, we wanted to determine whether a similar role could be observed 
in other model systems, such as cardiomyocytes derived from human iPSCs (hiPSCs). We therefore subjected human iPSC-derived Multipotent Cardiac Progenitors (MCPs)[44] to siRNAs against the human ortholog of $N a c \alpha, N A C A$, to assess their effects on cell proliferation and differentiation (Figure 5)[9]. We evaluated the propensity for MCPs to spontaneously differentiate into different cell types and calculated their relative proportions, by staining with $\alpha$-Actinin 1 (ACTN1) for cardiomyocytes (CM), Transgelin (TAGLN) for fibroblasts, and Cadherin 5 (CDH5) for endothelial cells. Total cell number was quantified by counting the number of DAPI-positive nuclei. The proportion of differentiated cell types was assessed nine days after siRNA treatment, a timepoint when active $\mathrm{KD}$ is no longer expected [9]. Efficiency of siRNA KD following transfection is expected to be maintained even when siRNAs against 3 different gene targets are combined, as demonstrated in previous experiments [7, 9].

Treatment of MCPs with NACA siRNA did not change total cell count compared to controls (Figure 5A,B), but significantly decreased the proportion of CMs (Figure 5C,D), and increased the proportion of fibroblast (Figure 5E,F). The proportion of endothelial cells remained unchanged (Supplemental Figure 5A,B). These results suggest that NACA may play a role in directing cell fate toward a cardiac program and its absence shifts these fates toward fibroblast differentiation.

We wondered whether the effect of NACA on CM differentiation can be similarly reversed by human orthologs of Drosophila $A b d-B$. Therefore, we tested the effect of siRNAs against human Homeobox C12 (HOXC12) or Homeobox D12 (HOXD12), which have close sequence homology with Abd-B. Treatment of either HOXC12 and HOXD12 siRNAs individually did not have a significant effect on total (Figure 5A), cardiomyocyte (Figure 5C), fibroblast (Figure 5E), or endothelial (Supplemental Figure 5A) cell populations compared to controls. When we tested combinations of NACA siRNA with HOXC12 and HOXD12 siRNA, or all three, we found that the Hox genes could alter NACA phenotypes. While treatment with NACA siRNA led to a reduced trend in total cell populations, combined KD of NACA and HOXC12 and HOXD12 led to total cell populations that were significant higher than NACA SiRNA treatment alone and were more similar to controls (Figure $\mathbf{5} \mathbf{A}, \mathbf{B}$ ). More striking is the reversal in the proportion of cardiomyocyte and fibroblast populations when NACA siRNA was combined with HOXC12 and HOXD12. Cotransfection of NACA with the selected Hox genes led to increased CM (Figure 5C,D) and reduced fibroblasts (Figure 5E,F) such that they are significantly different compared to NACA siRNA treatment alone and no longer different compared to controls. These results suggest that the reduction in $\mathrm{CM}$ and increase in fibroblast caused by NACA KD is dramatically rescued upon 
HOX co-KD, thus restoring CM differentiation of these pluripotent cells (Figure 5G). These results are consistent with observed cardiac differentiation and genetic interactions in Drosophila and that Naca/NACA activity in the heart may in part be mediated by posterior Hox genes.

As a positive control and a means of comparison, we transfected MCPs with siRNAs against cardiogenic transcription factors GATA Binding Protein 4 and 6 (GATA4/6) and Myocardin $(M Y O C D)[45,46]$. GATA4/6,MYOCD KD caused a significant decrease in total cell number (Figure 5A,B) and proportion of cardiomyocytes (Figure 5C,D), no change in the proportion of fibroblast (Figure 5E,F), and an increase in the proportion of endothelial cells (Supplemental Figure 5A,B). Thus, the effect of $N A C A$ KD on the proportion of cell types were different compared to the cardiogenic factors. The only similarity observed between NACA and GATA4/6,MYOCD KD was a decrease in the proportion of cardiomyocytes, although NACA produced a greater decrease (Figure 5 C,D). These results suggest that NACA KD induced alternate cell fates, possibly by a different mechanism to that of these cardiogenic transcription factors.

\section{Knockdown of individual SRP subunits cause distinct cardiac phenotypes}

NAC is just one of the protein biogenesis quality control mechanisms that is found at the ribosomal exit site sifting through emerging nascent polypeptides and guiding protein fates. SRP is another protein complex involved in controlling protein biogenesis by binding a disparate subset of nascent polypeptides destined for the ER, wherein its targeting is influenced by the activity of NAC. Therefore, we wanted to examine if disruption of SRP function in fly hearts would result in similar defects compared to Naca KD. Eukaryotic SRP is composed of a 7SL SRP RNA that holds the conformation of 6 proteins (SRP9, SRP14, SRP19, SRP54, SRP68, and SRP72) and targets approximately $30 \%$ of newly synthesized proteins to the ER (Figure 6A)[47]. SRP primarily recognizes the $\mathrm{N}$-terminal hydrophobic sequences of emerging nascent polypeptides, but has also been shown to bind nascent chains even when target sequences are not yet accessible [48]. In yeast, SRP also binds nascent chains with internal transmembrane domains [49]. Once bound, SRP arrests translation of the nascent chain until the SRP-ribosome complex binds with the SRPreceptor (SR) anchored to the ER, where translation of the nascent chain is restarted and cotranslationally released through the Sec61p translocase for insertion. 
Each subunit of the SRP complex exhibits specialized roles in the binding and translocation process (Figure 6A) and therefore, each subunit could confer specialization of SRP function through recruitment of cofactors or selective targeting of nascent proteins. We therefore knocked down each of the SRP subunits individually, as well as the $\beta$-subunit of SRP Receptor $(S R-\beta)$, to explore their role in heart development and how their phenotypes compare to NAC $\alpha$ and bic KD. RNAi mediated KD of either Srp9 or Srp14 subunits in the heart driven by Hand4.2GAL4 using two different RNAi lines did not produce gross differences in the heart structure compared to controls (Figure 6 B-D). In contrast, KD of Srp68 led to complete absence of the adult heart, similar to Naca KD (Figure 6E). Upon KD of Srp72, the heart tube was still present which is unlike the Naca KD phenotype. The very anterior segment, called the conical chamber,

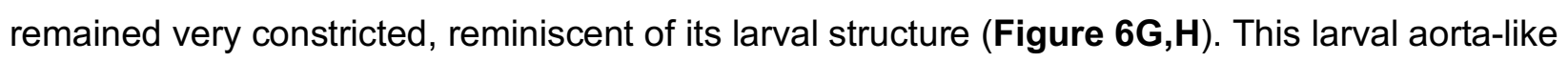
structure suggests that this section of the heart failed to undergo remodeling. KD of Srp19 led to a partial heart phenotype, where the anterior segment of the adult heart tube is absent, but retained a posterior segment, including the terminal chamber (Figure 6F), again unlike what was observed with Naca KD. KD of Srp54, which recognizes and binds the signal sequence on the nascent polypeptide, led to a malformed heart with missing cardiomyocytes in random positions throughout the tube (Figure $6 \mathbf{I , J}$ ). KD of $S R-\beta$ led to a heart tube with missing cardiomyocytes, which were most often the internal valves of the heart (Figure 6K-M). Interestingly, in vivo imaging of fluorescently labeled hearts in early pupae demonstrates that an intact larval heart tube forms with KD of any SRP components (Supplemental Figure 6), suggesting that their requirement prior to pupal remodeling of the heart may be less critical. Considering Srp19 and Srp68 KD largely abolished in adult hearts, the presence of an intact heart tube prior to cardiac remodeling in pupae suggests, that like $N A C \alpha$, their activity is critically required during cardiac remodeling and morphogenesis.

In summary, the KD of individual NAC and SRP subunits led to a range of distinct cardiac phenotypes that suggests each subunit and complex may function with distinct cell-type, regional and temporal specificities during cardiac development. Each subunit may contribute specialized function and interact with likely numerous cofactors that could target subsets of genes differently between SRP and NAC $\alpha$.

\section{Discussion}


Nascent polypeptide Associated Complex (NAC) and the Signal Recognition Particle (SRP) are integral to protein biogenesis. Our work suggests that they are pertinent for establishing distinct proteomic landscapes that shape cell identity and direct developmental fates (Figure 7). We demonstrated a developmental role for the NAC subunit Naca, in the Drosophila heart and Human Multipotent Cardiac Progenitors (MCPs) that influenced cardiac cell identity and morphogenesis through associations with Hox genes. These cardiac defects were triggered by knockdown (KD) of $\mathrm{Nac} \alpha$ at specific stages in development. Similarly, disruptions in SRP subunit expression led to cardiac cell type specific defects in the fly and some changes in heart morphology occurred during later, critical stages of development. These results suggest that Nac $\alpha$ and SRP function and selection of protein targets were temporally regulated. Components involved in protein translation are a growing family of genes associated with tissue-specific and developmental defects [50], including the heart [51, 52]. For example, patients with Diamond Blackfan Anemia (DBA), a bone marrow failure syndrome caused by ribosomopathies, exhibit higher manifestations of CHD compared to the general population [53]. In other cases, ribosomopathies led to CHD defects without overt hematological abnormalities [9, 54, 55]. Nac $\alpha$ specifically has been associated with Tetralogy of Fallot [11] and increased myocardial mass [10]. Along the SRP pathway, SRP54 mutations cause Schwachman-Diamond-like syndrome, a condition associated with CHDs [56, 57]. Furthermore, within the Pediatric Cardiac Genomic Consortium cohort [58], the SRP Receptor- $\alpha$ was associated with atrial septal defects while SRP Receptor- $\beta$ was associated with cyanotic congenital heart disease including ventricular septal defect. As genomic studies of patients uncover new variants associated with CHD, a closer examination of variants within translational genes and their co-occurrence with gene variants within developmental genetic networks is warranted. The question remains how these generic proteins can achieve both tissue specificity and temporal control in their translational functions that are critical for normal cardiac cell identity and fly heart tube morphogenesis.

Our studies found that KD of Nac $\alpha$ restricted by Hand4.2-GAL4 to mid- to late stages of embryo development (beginning stage 14 until hatching) specifically in cardiac relevant cells induced posterior terminal heart chamber defects that were evident in adult morphology (Figure 4 E,F). This suggests spatial specificity in Nac $\alpha$ function with embryonic origins targeting posterior cardiac cells more severely compared to more anterior regions. We also demonstrated that Nac $\alpha$ genetically interacts with a posteriorly expressed Hox gene, $A b d-B$, later during pupal stages, which further supports a stronger requirement for Nac $\alpha$ in the posterior heart; thus, heart defects 
occur from posterior to anterior regions with severity of $\mathrm{KD}$, i.e. in embryo or pupae vs. both (Figure 3). These regional differences in Nac $\alpha$ activity is consistent with a previously described role for NAC in the posterior patterning of the developing Drosophila embryo [19, 20]. In the early syncytial embryo, posterior patterning proteins, such as OSKAR and NANOS, accumulate in the posterior end of the embryo. Components of the NAC complex were necessary to restrict translation and localization of OSKAR and NANOS to the posterior end [19, 20]. Absence of NAC components resulted in ectopic expression of posterior patterning proteins anteriorly leading to a lethal, bicaudal phenotype. How this translational control is achieved remains unclear, but could involve targeted translational pausing either through 3'UTR sequences or additional translation cofactors. In our work, the posterior preference is retained in an embryo where Nac $\alpha$ is knocked down only in the developing heart cells, which supports the idea that additional cofactors with posterior-anterior specific expression and/or function contribute to regulate Nac $\alpha$ activity.

Future studies will be aimed at determining the mechanisms that regulate Nac $\alpha$ activity that drives these anterior-posterior specific responses in the heart. Furthermore, how Nac $\alpha$ regulates Hox gene $A b d-B$ repression in the anterior heart regions, and possibly other cardiac relevant targets, could help uncover pathways leading to targeted tissue defects and corresponding human diseases. Our data show that KD of Nac $\alpha$ is permitting $A b d-B$ protein expression in the anterior regions of the larval heart and aorta, where it is normally absent (Figure 3 C-E). Ectopic Abd-B expression could occur through direct disinhibition of Nac $\alpha$ translational control of $A b d-B m R N A$. Alternatively, Nac $\alpha$ KD could have an indirect effect on cell identity that aberrantly turns on $A b d-B$ transcription and subsequent protein expression. In addition, $A b d-B$ protein is no longer strictly nuclear, rather is mistargeted and expressed throughout the cardiac cell of Naca KD flies (Figure 3E). This could in part be a result of nascent protein mistargeting toward the SRP pathway that redirect $A b d-B$ to the ER as well as secreted into the lumen. Hox genes play a crucial role in early cardiac specification, patterning and remodeling in the heart [59] and Nac $\alpha$ translational regulation could be a novel mechanism of Hox gene regulation that may contribute to pathogenesis of CHD.

Within the mammalian heart a similar role for regulated anterior-posterior hox gene expression is crucial for proper morphogenesis. Complex signaling events coordinate patterning of cardiac progenitor populations within the First and Second Heart Fields (SHF) [60]. Along the anterior and posterior axis of the SHF, cardiac progenitor cells are further subdivided into domains 
that express distinct transcriptional programs contributing to the development of distinct heart structures [61]. Characterization of the transcriptional profiles within subdomains have implicated specific Hox gene expression (i.e. Hoxa1 and Hoxb1) to establish cellular identity and cell fate $[61,62]$. Future work in mammals can help determine whether NAC and SRP activity may contribute to targeted expression of Hox genes within distinct subdomains of the SHF, which is suggested by our work in flies and human MCPs.

The effect of $\mathrm{Nac} \alpha \mathrm{KD}$ in fly hearts has a number of parallels to the targeted developmental mishaps resulting from mutation of another ubiquitous translational ribosomal protein, $R p / 38$, in mice. These mutant mice display specific axial skeletal patterning defects attributable to reduced association of a specific subset of Hox mRNA with polysomes (Hox a4; a5; a9; a11; b3; b13; c8; d10), which led to reduced protein levels of these Hox (validated for HOXA5, HOXA11 HOXB13 proteins), without changes in global protein translation [63, 64]. Mice with loss-of-function mutations in the targeted Hox genes (Hoxa5, Hoxa9, Hoxa11, Hoxc8, Hoxd10) phenocopy numerous aspects of the Rp/38 mutant phenotypes along the rostro-caudal axis, suggesting that the altered translation of these Hox genes inflicted by Rp/38 deficiency mediated the skeletal patterning defects. The results in flies and mice support the idea that seemingly generic components of the protein biogenesis pathway can impose a layer of translational control pertinent to tissue-specific development.

It is remarkable that the complete histolysis of the adult fly heart observed during pupal stages required KD of Naca during both embryonic and pupal stages, either one alone was not sufficient (Figure 4 D-H). This was surprising, since during pupal stages the heart undergoes extensive remodeling, which requires significant protein biogenesis, and pupal-only KD of Nac $\alpha$ does not induce cell death, nor significant changes in heart structure or function. This suggests that Nac $\alpha$ function during embryonic stages may create a proteomic landscape establishing longterm programming of cardiomyocyte identity properly poised for future developmental responses, such as remodeling to the adult heart during metamorphosis. An embryonic requirement for later cardiac remodeling and survival was also found for ribosomal protein gene $R p L 13$ [9]. However, in the case for $R p L 13, K D$ in the embryo alone resulted in significant heart loss during pupal stages. Thus, again translational perturbances in the embryo can have long-lasting effects, suggesting a mechanism by which preprograming in the embryonic heart is critical for appropriate cardiac remodeling later in development. How translational proteins and mechanisms contribute to redefining cell identity would need to be better understood. 
Our work using MCPs demonstrated a role for Nac $\alpha$ in human cardiac cell specification and differentiation. KD of Nac $\alpha$ redirected progenitor cell fates from a cardiomyocyte lineage toward a fibroblast lineage (Figure 5). In contrast, KD of cardiogenic transcription factors Gata4/6 and $M y o C D$, directed progenitor cell fates toward a different population profile with decreased total cell population and decreased cardiomyocytes, but increased endothelial cells and no change in fibroblasts (Figure 5) [65,66]. Although this difference in population profiles induced by KD of Nac $\alpha$ vs cardiogenic transcription factors suggests that Nac $\alpha$ utilizes distinct mechanisms for refinement of cardiac cell fates, it remains to be determined how these processes are coordinated.

Defects in differentiation and maturation caused by Nac $\alpha$ deficiency in flies are also observed in other cell lines and model systems including deviations in cell fates of osteoblast, myotubes and haematopoietic stem/progenitor cells $[24,26]$. In higher order organisms, Nac $\alpha$ has evolved to encompass additional isoform-specific functions, including as a transcriptional coactivator [67]. Furthermore, a larger, alternatively spliced isoform of $N a c \alpha$ (skNAC), containing an additional proline-rich exon, is expressed specifically in striated muscle of vertebrates [68] and plays a role in transcriptional regulation, fiber-type specification, myofibrillogenesis, and sarcomerogenesis $[27,28,31,32,68,69]$. This puts in question whether in flies Nac $\alpha$ only functions in translational regulation or may be involved in other cellular activities.

Protein biogenesis and its regulation at the ribosome exit site relies on a network of nascent protein chaperones. Because of the interactions between NAC and SRP that refine their ability to target specific nascent polypeptides emerging from ribosomes, we wanted to explore the effects of disrupting SRP activity on fly heart development and compare them to Nac $\alpha \mathrm{KD}$. We found that KD of individual SRP subunits led to unique cardiac phenotypes (Figure 6). While Srp9 and Srp14, responsible for elongation arrest during translocation, did not alter adult heart structure, KD of Srp68, responsible for docking to the SR receptor on the ER membrane, led to complete loss of the heart. The subunit Srp72 binds to Srp68 and participates in SR docking activity, but its KD did not result in complete heart loss, but the defect was focused on the conical chamber of the heart, possibly hindering larval aorta transdifferentation into the adult fly heart during metamorphosis. KD of Srp54, a highly evolutionarily conserved protein subunit that bind to signal sequences on nascent polypeptides, led to missing cardiomyocytes at random positions 
in the heart. KD of Srp19, responsible for linking Srp54 to the rest of the SRP complex led to a partial heart loss anteriorly, in contrast to Nac $\alpha$, which preferentially deleted posterior portions of the heart. KD of the $\beta$ subunit of the SR that binds nascent protein-SRP-ribosome complex at the ER membrane, led to dysmorphic cardiomyocytes, which were usually the internal valve regions. The varied phenotypes suggest specialized roles and a complexity in spatial regulation for each SRP subunit, similar to the posterior preference seen for Nac $\alpha$ KD. Furthermore, SRP's influence on cardiogenesis is temporally regulated, as gross changes in morphology occur during crucial developmental stages, such as the disappearance of the heart during metamorphosis induced by Srp68 KD (Figure 6E, Supplemental Figure 6).

Determining how KD of SRP subunits display cardiac tissue-specific effects would uncover novel aspects of protein biogenesis control in a cell-type specific manner. In contrast to Naca, the SRP complex interacts with nascent proteins that are destined for insertion into the rough ER and subsequent translocation to the plasma membrane or for secretion. Therefore, SRP targets are likely enriched for membrane receptors. Thus, interruption of receptor function and signaling mediating non-cell autonomous cues may be partially involved in the cardiac phenotypes caused by disruption of SRP activity. For example, cardiac cells may respond aberrantly to ecdysone steroid hormone secreted by the ring gland during metamorphosis due to a lack of Ecdysone Receptor expression at the cardiac cell membrane. Indeed, this may in part be a contributing mechanism, as Srp72 KD in the heart resulted in constriction of just the conical chamber in the anterior region of the adult heart, which resembled an unremodeled larval heart (Figure 6G,H). Still, why there appears to be regional specificity to this lack of Ecdysone Receptor response, despite Srp72 KD throughout the heart, remains to be determined. Furthermore, KD of each SRP subunit resulted in differential effects on heart morphology that target discrete heart regions and cell types, suggesting a mechanism for tissue-specific regulation. It is possible that each SRP subunit binds a disparate set of differentially expressed cofactors to impart specific functional control of SRP activity.

In summary, our work demonstrated specific roles for the NAC and the SRP complex in cardiac development, using Drosophila and human MCPs, and offers novel mechanisms in the regulation of the cardiac proteome to establish cell identity and tissue patterning (Figure 7). How generic components of the translational machinery could lead to targeted effects on heart development and function is likely layered in a complex process that remains to be explored. 


\section{METHODS}

\section{Drosophila Strains}

Heart-specific control of transcription was achieved by the GAL4-UAS system [70], in which the following cardiac relevant GAL4 drivers were used: Hand4.2-GAL4 [33, 71, 72], tinC $\Delta 4$ GAL4 [73], tinD-GAL4 [74], and tinHE-GAL4 [75]. Dot-GAL4 driver line [34] was used to express in pericardial cells. Drosophila GD and KK RNAi collection lines along with appropriate controls were obtained from the Vienna Drosophila Resource Center (VDRC)[76]. Two copies of a temperature-sensitive tubulin-GAL80 (tubulin-GAL80 ${ }^{\text {ts }}$ ) were recombined and combined with the Hand4.2-GAL4 driver (Hand4.2-GAL4, Tubulin-GAL80 ${ }^{\text {ts; }}$ Tubulin-GAL80 ${ }^{\text {ts }}$, HTT; [9]). All fly lines are listed in Supplemental Table 1.

\section{Developmental Regulation of Gene Expression}

The HTT line allows for transcriptional control of constructs fused to a UAS enhancer by manipulation of ambient temperature. At $18^{\circ} \mathrm{C}$, GAL80 is intact and blocks GAL4 mediated activation of the UAS enhancer. A shift to $29^{\circ} \mathrm{C}$ destabilizes GAL80 protein, permitting GAL4 binding to the UAS enhancer to induce transcription. As control, flies were placed in $18^{\circ} \mathrm{C}$ or $28^{\circ} \mathrm{C}$ throughout development until eclosion when an intact or an absent heart were expected, respectively. For Nac $\alpha$ knockdown (KD) in embryonic stages, female and male flies were placed in $29^{\circ} \mathrm{C}$ and embryos collected every 2 hours. Embryos were maintained in $29^{\circ} \mathrm{C}$ for 24 hours and then shifted to $18^{\circ} \mathrm{C}$ for the remainder of development. Another set of embryos were maintained in $29^{\circ} \mathrm{C}$ for 48 hours before shifting to $18^{\circ} \mathrm{C}$. For Nac $\alpha$ KD during pupal stages, fly crosses were maintained in $18^{\circ} \mathrm{C}$ until wandering $3^{\text {rd }}$ instar larvae were collected. They were then immediately placed in $29^{\circ} \mathrm{C}$ until eclosion. For KD of Nac $\alpha$ starting at mid-larvae, embryos from fly crosses at $18^{\circ} \mathrm{C}$ were collected every 2 hours, maintained in $18^{\circ} \mathrm{C}$ for 16 days when larvae were approximately at L2 stages. Larvae were then placed in $29^{\circ} \mathrm{C}$ until eclosion. Finally, to test $\operatorname{Nac} \alpha$ $\mathrm{KD}$ during embryonic and pupal stages only, embryos were collected from fly crosses at $29^{\circ} \mathrm{C}$ for 24 hours as described above, and then returned to $18^{\circ} \mathrm{C}$ to develop through larval stages. Wandering larvae were then collected and then placed in $29^{\circ} \mathrm{C}$ until eclosion. Adult flies were assessed for heart function using SOHA (see below) and immunostained to examine structure (see Figure 4). 


\section{Immunostaining of Adult Drosophila Hearts}

Adult flies were dissected and treated with 10mM EGTA in PBT (PBS + Triton-X-100; $0.03 \%$ Triton $X-100$ ), for 2 minutes to maintain a relaxed state of the heart. Hearts were then fixed with 4\% PFA in PBT for 20 minutes, followed by three 10-minute PBT washes. Hearts were stained with primary antibodies (EC11 Pericardin, Developmental Studies Hybridoma Bank, DSHB) and incubated overnight in $4^{\circ} \mathrm{C}$. Hearts were then washed with PBT three times for 15 minutes each, followed by incubation with fluorescent secondary antibodies (1:500, Jackson ImmunoResearch Laboratories, Inc.) and Alexa Fluor conjugated phalloidin (1:300, Life Technologies) in $4^{\circ} \mathrm{C}$ overnight. Hearts were then washed with PBT three times for 15 minutes each and then once with PBS. Hearts were mounted using ProLong Gold Mountant with DAPI (Life Technologies). Immunostained preparations were visualized with an Imager.Z1 equipped with an Apotome2 (Carl Zeiss, Jena), Hammamatsu Orca Flash4.0 camera, and ZEN imaging software (Carl Zeiss).

\section{Live-imaging of the Heart in Drosophila Pupae}

For fluorescence-based heart function analysis, we crossed a fly line that contained both Hand4.2-GAL4 and a heart enhancer fused to tandem-Tomato fluorescent protein (tdtK), to controls, Nac $\alpha$-RNAi, or bicaudal-RNAi flies [76]. White pupae (WP) from these crosses were collected and lined up on small petridishes with dorsal side facing up. A subset of WP was imaged immediately while another subset was aged for approximately 20 hours in room temperature. Dishes were placed on a Zeiss Imager M1 equipped with a Hamamatsu Orca-Flash 4.0 Digital Camera (C11440), and heart images were captured every 2 minutes, for a duration of about 55 hours at room temperature (approx. $20^{\circ} \mathrm{C}$ ) using ZEN imaging software (Carl Zeiss). Movies were formatted and compiled using ImageJ and iMovie (Apple).

\section{Heart Function Analysis}

Assessment of Drosophila heart function and structure using the Semi-automatic Optical Heartbeat Analysis (SOHA) method as previously described [77, 78]. Briefly, four-day old adult flies were anaesthetized with FlyNap (Carolina Biological Supply Co, Burlington, NC) and dissected in oxygenated artificial hemolymph to expose the beating heart within the abdomen. Hearts were placed on an Olympus BX61WI microscope while being filmed through a 10x water immersion lens with a high-speed digital camera (Hamamatsu Photonics C9300 digital camera) 
using $\mathrm{HCl}$ image capture software (Hamamatsu). High-speed movies were analyzed using the Semi-automated Optical Heartbeat Analysis (SOHA) software [77, 78]. Parameters measured include Heart Period (HP), Diastolic Interval (DI), Systolic Interval (SI), Arrhythmicity Index (AI), Diastolic Diameter (DD), Systolic Diameter (SD) and Fractional Shortening (FS) (Figure 2A). FS, a measure of contractility, is calculated using the following equation $F S=(D D-S D) / D D$.

\section{MCP cell culture, siRNA transfection, and Immunostaining}

A pool of 4 unique siRNA sequences targeting different regions of selected genes and random control were obtained from the human siGENOME library from Dharmacon, Inc. Frozen 5-day old human MCPS [44, 79] were thawed and transfected with siRNAs at 5nM final concentration using Lipofectamine RNAiMAX transfection reagent (Invitrogen). Approximately 20,000 cells were plated in each well of a 384-well plate (Greiner Bio-One) coated with Matrigel Basement Membrane Matrix (Corning). Cells were incubated at $37^{\circ} \mathrm{C}$ and media refreshed every second day. Each experiment contained quadruplicate technical replicates per condition and performed on different batches of MCP clones programmed independently.

Immunostaining to identify cell-type composition of cultures were performed 9 days following siRNA transfection. Cells were fixed with warmed 4\% paraformaldehyde solution for 30 minutes without agitation, followed by an additional 30 minutes of fixing with agitation. Wells were washed with Phosphate Buffered Saline (PBS) for 10 mins and repeated three times. Samples were then incubated with blocking solution for 30 mins (10\% horse serum, $2.5 \%$ Triton-X-100, $10 \%$ gelatin). Primary antibodies were diluted in blocking solution and added to samples for 1 hour at room temperature (RT): ACTN1 (Sigma, A7811), TAGLN (Abcam, Ab14106), CDH5 (R\&D Systems, AF938). Wells were washed with PBS for 15 mins and repeated three times. Samples were incubated with Alexa-conjugated secondary antibodies (Life Technologies) including DAPI diluted in blocking solution for 1 hour at RT. Wells were washed for 15 minutes and repeated three times, following which samples were imaged using a High-Throughput microscope (ImageXpress, Molecular Devices). Fluorescence was quantified using custom MetaXpress software (Molecular Devices) whereby, the number of total cells and cells positive for ACTN1, TAGLN, and CDH5 in each sample were quantified [44, 79].

\section{Statistical Analysis}

Statistical analysis was performed using GraphPad Prism (GraphPad Software, La Jolla USA). Data are presented as the mean \pm SEM. Human MCP, cardiomyocyte and fibroblast data 
were analyzed using a one-way ANOVA analysis followed by a Dunnett's multiple comparison post-hoc test. A student's unpaired t-test was used to analyze Drosophila heart function data.

\section{References}

1. van der Bom, T., Zomer, A.C., Zwinderman, A.H., Meijboom, F.J., Bouma, B.J., and Mulder, B.J. (2011). The changing epidemiology of congenital heart disease. Nat. Rev. Cardiol. 8, 50-60.

2. Williams, K., Carson, J., and Lo, C. (2019). Genetics of Congenital Heart Disease. Biomolecules 9.

3. Zaidi, S., and Brueckner, M. (2017). Genetics and Genomics of Congenital Heart Disease. Circ. Res. 120, 923-940.

4. Pierpont, M.E., Brueckner, M., Chung, W.K., Garg, V., Lacro, R.V., McGuire, A.L., Mital, S., Priest, J.R., Pu, W.T., Roberts, A., et al. (2018). Genetic Basis for Congenital Heart Disease: Revisited: A Scientific Statement From the American Heart Association. Circulation 138, e653-e711.

5. Lage, K., Greenway, S.C., Rosenfeld, J.A., Wakimoto, H., Gorham, J.M., Segre, A.V., Roberts, A.E., Smoot, L.B., Pu, W.T., Pereira, A.C., et al. (2012). Genetic and environmental risk factors in congenital heart disease functionally converge in protein networks driving heart development. Proc. Natl. Acad. Sci. U. S. A. 109, 14035-14040.

6. Oyen, N., Poulsen, G., Boyd, H.A., Wohlfahrt, J., Jensen, P.K., and Melbye, M. (2009). Recurrence of congenital heart defects in families. Circulation 120, 295-301.

7. Theis, J.L., Vogler, G., Missinato, M.A., Li, X., Nielsen, T., Zeng, X.I., Martinez-Fernandez, A., Walls, S.M., Kervadec, A., Kezos, J.N., et al. (2020). Patient-specific genomics and cross-species functional analysis implicate LRP2 in hypoplastic left heart syndrome. Elife 9.

8. Paige, S.L., Galdos, F.X., Lee, S., Chin, E.T., Ranjbarvaziri, S., Feyen, D.A.M., Darsha, A.K., Xu, S., Ryan, J.A., Beck, A.L., et al. (2020). Patient-Specific Induced Pluripotent Stem Cells Implicate Intrinsic Impaired Contractility in Hypoplastic Left Heart Syndrome. Circulation 142, 1605-1608.

9. Schroeder, A.M., Allahyari, M., Vogler, G., Missinato, M.A., Nielsen, T., Yu, M.S., Theis, J.L., Larsen, L.A., Goyal, P., Rosenfeld, J.A., et al. (2019). Model system identification of novel congenital heart disease gene candidates: focus on RPL13. Hum. Mol. Genet. 28, 3954-3969.

10. van der Harst, P., van Setten, J., Verweij, N., Vogler, G., Franke, L., Maurano, M.T., Wang, X., Mateo Leach, I., Eijgelsheim, M., Sotoodehnia, N., et al. (2016). 52 Genetic Loci Influencing Myocardial Mass. J. Am. Coll. Cardiol. 68, 1435-1448.

11. Liu, L., Wang, H.D., Cui, C.Y., Qin, Y.Y., Fan, T.B., Peng, B.T., Zhang, L.Z., and Wang, C.Z. (2017). Whole exome sequencing identifies novel mutation in eight Chinese children with isolated tetralogy of Fallot. Oncotarget 8, 106976-106988.

12. Wang, S., Sakai, H., and Wiedmann, M. (1995). NAC covers ribosome-associated nascent chains thereby forming a protective environment for regions of nascent chains just emerging from the peptidyl transferase center. J. Cell Biol. 130, 519-528. 
13. George, R., Beddoe, T., Landl, K., and Lithgow, T. (1998). The yeast nascent polypeptideassociated complex initiates protein targeting to mitochondria in vivo. Proc. Natl. Acad. Sci. U. S. A. 95, 2296-2301.

14. George, R., Walsh, P., Beddoe, T., and Lithgow, T. (2002). The nascent polypeptideassociated complex (NAC) promotes interaction of ribosomes with the mitochondrial surface in vivo. FEBS Lett. 516, 213-216.

15. Funfschilling, U., and Rospert, S. (1999). Nascent polypeptide-associated complex stimulates protein import into yeast mitochondria. Mol. Biol. Cell 10, 3289-3299.

16. Hsieh, H.H., Lee, J.H., Chandrasekar, S., and Shan, S.O. (2020). A ribosome-associated chaperone enables substrate triage in a cotranslational protein targeting complex. Nat Commun 11, 5840.

17. Saraogi, I., and Shan, S.O. (2011). Molecular mechanism of co-translational protein targeting by the signal recognition particle. Traffic 12, 535-542.

18. Zhang, Y., Berndt, U., Golz, H., Tais, A., Oellerer, S., Wolfle, T., Fitzke, E., and Rospert, S. (2012). NAC functions as a modulator of SRP during the early steps of protein targeting to the endoplasmic reticulum. Mol. Biol. Cell 23, 3027-3040.

19. Braat, A.K., Yan, N., Arn, E., Harrison, D., and Macdonald, P.M. (2004). Localizationdependent oskar protein accumulation; control after the initiation of translation. Dev. Cell 7, 125-131.

20. Markesich, D.C., Gajewski, K.M., Nazimiec, M.E., and Beckingham, K. (2000). bicaudal encodes the Drosophila beta NAC homolog, a component of the ribosomal translational machinery*. Development 127, 559-572.

21. Smith, J.L., Wilson, J.E., and Macdonald, P.M. (1992). Overexpression of oskar directs ectopic activation of nanos and presumptive pole cell formation in Drosophila embryos. Cell 70, 849-859.

22. Ephrussi, A., and Lehmann, R. (1992). Induction of germ cell formation by oskar. Nature 358, 387-392.

23. St-Arnaud, R., and Quelo, I. (1998). Transcriptional coactivators potentiating AP-1 function in bone. Front. Biosci. 3, d838-848.

24. Moreau, A., Yotov, W.V., Glorieux, F.H., and St-Arnaud, R. (1998). Bone-specific expression of the alpha chain of the nascent polypeptide-associated complex, a coactivator potentiating c-Jun-mediated transcription. Mol. Cell. Biol. 18, 1312-1321.

25. Hekmatnejad, B., Mandic, V., Yu, V.W., Akhouayri, O., Arabian, A., and St-Arnaud, R. (2014). Altered gene dosage confirms the genetic interaction between FIAT and alphaNAC. Gene 538, 328-333.

26. Murayama, E., Sarris, M., Redd, M., Le Guyader, D., Vivier, C., Horsley, W., Trede, N., and Herbomel, P. (2015). NACA deficiency reveals the crucial role of somite-derived stromal cells in haematopoietic niche formation. Nat Commun 6, 8375.

27. Li, H., Randall, W.R., and Du, S.J. (2009). skNAC (skeletal Naca), a muscle-specific isoform of Naca (nascent polypeptide-associated complex alpha), is required for myofibril organization. FASEB J. 23, 1988-2000.

28. Berger, F., Berkholz, J., Breustedt, T., Ploen, D., and Munz, B. (2012). Skeletal musclespecific variant of nascent polypeptide associated complex alpha (skNAC): implications for a specific role in mammalian myoblast differentiation. Eur. J. Cell Biol. 91, 150-155. 
29. Berkholz, J., Orgeur, M., Stricker, S., and Munz, B. (2015). skNAC and Smyd1 in transcriptional control. Exp. Cell Res. 336, 182-191.

30. Berkholz, J., Michalick, L., and Munz, B. (2014). The E3 SUMO ligase Nse2 regulates sumoylation and nuclear-to-cytoplasmic translocation of skNAC-Smyd1 in myogenesis. J. Cell Sci. 127, 3794-3804.

31. Park, C.Y., Pierce, S.A., von Drehle, M., Ivey, K.N., Morgan, J.A., Blau, H.M., and Srivastava, D. (2010). skNAC, a Smyd1-interacting transcription factor, is involved in cardiac development and skeletal muscle growth and regeneration. Proc. Natl. Acad. Sci. U. S. A. 107, 20750-20755.

32. Sims, R.J., 3rd, Weihe, E.K., Zhu, L., O'Malley, S., Harriss, J.V., and Gottlieb, P.D. (2002). $\mathrm{m}$-Bop, a repressor protein essential for cardiogenesis, interacts with skNAC, a heartand muscle-specific transcription factor. J. Biol. Chem. 277, 26524-26529.

33. Han, Z., Yi, P., Li, X., and Olson, E.N. (2006). Hand, an evolutionarily conserved bHLH transcription factor required for Drosophila cardiogenesis and hematopoiesis. Development 133, 1175-1182.

34. Kimbrell, D.A., Hice, C., Bolduc, C., Kleinhesselink, K., and Beckingham, K. (2002). The Dorothy enhancer has Tinman binding sites and drives hopscotch-induced tumor formation. Genesis 34, 23-28.

35. Monier, B., Astier, M., Semeriva, M., and Perrin, L. (2005). Steroid-dependent modification of Hox function drives myocyte reprogramming in the Drosophila heart. Development 132, 5283-5293.

36. Ponzielli, R., Astier, M., Chartier, A., Gallet, A., Therond, P., and Semeriva, M. (2002). Heart tube patterning in Drosophila requires integration of axial and segmental information provided by the Bithorax Complex genes and hedgehog signaling. Development 129, 4509-4521.

37. Lo, P.C., and Frasch, M. (2003). Establishing A-P polarity in the embryonic heart tube: a conserved function of Hox genes in Drosophila and vertebrates? Trends Cardiovasc. Med. 13, 182-187.

38. Lo, P.C., Skeath, J.B., Gajewski, K., Schulz, R.A., and Frasch, M. (2002). Homeotic genes autonomously specify the anteroposterior subdivision of the Drosophila dorsal vessel into aorta and heart. Dev. Biol. 251, 307-319.

39. Lovato, T.L., Nguyen, T.P., Molina, M.R., and Cripps, R.M. (2002). The Hox gene abdominal-A specifies heart cell fate in the Drosophila dorsal vessel. Development 129, 5019-5027.

40. McGuire, S.E., Le, P.T., Osborn, A.J., Matsumoto, K., and Davis, R.L. (2003). Spatiotemporal rescue of memory dysfunction in Drosophila. Science 302, 1765-1768.

41. Gamerdinger, M., Hanebuth, M.A., Frickey, T., and Deuerling, E. (2015). The principle of antagonism ensures protein targeting specificity at the endoplasmic reticulum. Science 348, 201-207.

42. Kirstein-Miles, J., Scior, A., Deuerling, E., and Morimoto, R.I. (2013). The nascent polypeptide-associated complex is a key regulator of proteostasis. EMBO J. 32, 14511468. 
43. Arsenovic, P.T., Maldonado, A.T., Colleluori, V.D., and Bloss, T.A. (2012). Depletion of the C. elegans NAC engages the unfolded protein response, resulting in increased chaperone expression and apoptosis. PLoS One 7, e44038.

44. Cunningham, T.J., Yu, M.S., McKeithan, W.L., Spiering, S., Carrette, F., Huang, C.T., Bushway, P.J., Tierney, M., Albini, S., Giacca, M., et al. (2017). Id genes are essential for early heart formation. Genes Dev. 31, 1325-1338.

45. Pikkarainen, S., Tokola, H., Kerkela, R., and Ruskoaho, H. (2004). GATA transcription factors in the developing and adult heart. Cardiovasc. Res. 63, 196-207.

46. Pipes, G.C., Creemers, E.E., and Olson, E.N. (2006). The myocardin family of transcriptional coactivators: versatile regulators of cell growth, migration, and myogenesis. Genes Dev. 20, 1545-1556.

47. Akopian, D., Shen, K., Zhang, X., and Shan, S.O. (2013). Signal recognition particle: an essential protein-targeting machine. Annu. Rev. Biochem. 82, 693-721.

48. Mercier, E., Holtkamp, W., Rodnina, M.V., and Wintermeyer, W. (2017). Signal recognition particle binds to translating ribosomes before emergence of a signal anchor sequence. Nucleic Acids Res. 45, 11858-11866.

49. Schibich, D., Gloge, F., Pohner, I., Bjorkholm, P., Wade, R.C., von Heijne, G., Bukau, B., and Kramer, G. (2016). Global profiling of SRP interaction with nascent polypeptides. Nature 536, 219-223.

50. Armistead, J., and Triggs-Raine, B. (2014). Diverse diseases from a ubiquitous process: the ribosomopathy paradox. FEBS Lett. 588, 1491-1500.

51. Long, P.A., Theis, J.L., Shih, Y.H., Maleszewski, J.J., Abell Aleff, P.C., Evans, J.M., Xu, X., and Olson, T.M. (2017). Recessive TAF1A mutations reveal ribosomopathy in siblings with end-stage pediatric dilated cardiomyopathy. Hum. Mol. Genet. 26, 2874-2881.

52. Ward, T., Tai, W., Morton, S., Impens, F., Van Damme, P., Van Haver, D., Timmerman, E., Venturini, G., Zhang, K., Jang, M.Y., et al. (2021). Mechanisms of Congenital Heart Disease Caused by NAA15 Haploinsufficiency. Circ. Res. 128, 1156-1169.

53. Reller, M.D., Strickland, M.J., Riehle-Colarusso, T., Mahle, W.T., and Correa, A. (2008). Prevalence of congenital heart defects in metropolitan Atlanta, 1998-2005. J. Pediatr. $153,807-813$.

54. Vlachos, A., Osorio, D.S., Atsidaftos, E., Kang, J., Lababidi, M.L., Seiden, H.S., Gruber, D., Glader, B.E., Onel, K., Farrar, J.E., et al. (2018). Increased Prevalence of Congenital Heart Disease in Children With Diamond Blackfan Anemia Suggests Unrecognized Diamond Blackfan Anemia as a Cause of Congenital Heart Disease in the General Population: A Report of the Diamond Blackfan Anemia Registry. Circ Genom Precis Med 11, e002044.

55. Jin, S.C., Homsy, J., Zaidi, S., Lu, Q., Morton, S., DePalma, S.R., Zeng, X., Qi, H., Chang, W., Sierant, M.C., et al. (2017). Contribution of rare inherited and de novo variants in 2,871 congenital heart disease probands. Nat. Genet. 49, 1593-1601.

56. Bezzerri, V., and Cipolli, M. (2019). Shwachman-Diamond Syndrome: Molecular Mechanisms and Current Perspectives. Mol. Diagn. Ther. 23, 281-290.

57. Hauet, Q., Beaupain, B., Micheau, M., Blayo, M., Gandemer, V., Gottrand, F., Blin, N., Fouyssac, F., Lethor, J.P., Bellanne-Chantelot, C., et al. (2013). Cardiomyopathies and congenital heart diseases in Shwachman-Diamond syndrome: a national survey. Int. J. Cardiol. 167, 1048-1050. 
58. Hoang, T.T., Goldmuntz, E., Roberts, A.E., Chung, W.K., Kline, J.K., Deanfield, J.E., Giardini, A., Aleman, A., Gelb, B.D., Mac Neal, M., et al. (2018). The Congenital Heart Disease Genetic Network Study: Cohort description. PLoS One 13, e0191319.

59. Roux, M., and Zaffran, S. (2016). Hox Genes in Cardiovascular Development and Diseases. J Dev Biol 4.

60. Buckingham, M., Meilhac, S., and Zaffran, S. (2005). Building the mammalian heart from two sources of myocardial cells. Nat Rev Genet 6, 826-835.

61. Bertrand, N., Roux, M., Ryckebusch, L., Niederreither, K., Dolle, P., Moon, A., Capecchi, M., and Zaffran, S. (2011). Hox genes define distinct progenitor sub-domains within the second heart field. Dev. Biol. 353, 266-274.

62. Stefanovic, S., Laforest, B., Desvignes, J.P., Lescroart, F., Argiro, L., Maurel-Zaffran, C., Salgado, D., Plaindoux, E., De Bono, C., Pazur, K., et al. (2020). Hox-dependent coordination of mouse cardiac progenitor cell patterning and differentiation. Elife 9.

63. Kondrashov, N., Pusic, A., Stumpf, C.R., Shimizu, K., Hsieh, A.C., Ishijima, J., Shiroishi, T., and Barna, M. (2011). Ribosome-mediated specificity in Hox mRNA translation and vertebrate tissue patterning. Cell 145, 383-397.

64. Shi, Z., Fujii, K., Kovary, K.M., Genuth, N.R., Rost, H.L., Teruel, M.N., and Barna, M. (2017). Heterogeneous Ribosomes Preferentially Translate Distinct Subpools of mRNAs Genome-wide. Mol. Cell 67, 71-+.

65. Maliken, B.D., Kanisicak, O., Karch, J., Khalil, H., Fu, X., Boyer, J.G., Prasad, V., Zheng, Y., and Molkentin, J.D. (2018). Gata4-Dependent Differentiation of c-Kit(+)-Derived Endothelial Cells Underlies Artefactual Cardiomyocyte Regeneration in the Heart. Circulation 138, 1012-1024.

66. Masino, A.M., Gallardo, T.D., Wilcox, C.A., Olson, E.N., Williams, R.S., and Garry, D.J. (2004). Transcriptional regulation of cardiac progenitor cell populations. Circ. Res. 95, 389-397.

67. Yotov, W.V., Moreau, A., and St-Arnaud, R. (1998). The alpha chain of the nascent polypeptide-associated complex functions as a transcriptional coactivator. Mol. Cell. Biol. 18, 1303-1311.

68. Yotov, W.V., and St-Arnaud, R. (1996). Differential splicing-in of a proline-rich exon converts alphaNAC into a muscle-specific transcription factor. Genes Dev. 10, 17631772.

69. Berkholz, J., Zakrzewicz, A., and Munz, B. (2013). skNAC depletion stimulates myoblast migration and perturbs sarcomerogenesis by enhancing calpain 1 and 3 activity. Biochem. J. 453, 303-310.

70. Brand, A.H., and Perrimon, N. (1993). Targeted gene expression as a means of altering cell fates and generating dominant phenotypes. Development 118, 401-415.

71. Hallier, B., Hoffmann, J., Roeder, T., Togel, M., Meyer, H., and Paululat, A. (2015). The bHLH Transcription Factor Hand Regulates the Expression of Genes Critical to Heart and Muscle Function in Drosophila melanogaster. PLoS One 10, e0134204.

72. Togel, M., Meyer, H., Lehmacher, C., Heinisch, J.J., Pass, G., and Paululat, A. (2013). The bHLH transcription factor hand is required for proper wing heart formation in Drosophila. Dev. Biol. 381, 446-459. 
73. Lo, P.C., and Frasch, M. (2001). A role for the COUP-TF-related gene seven-up in the diversification of cardioblast identities in the dorsal vessel of Drosophila. Mech. Dev. 104, 49-60.

74. Yin, Z., Xu, X.L., and Frasch, M. (1997). Regulation of the twist target gene tinman by modular cis-regulatory elements during early mesoderm development. Development 124, 4971-4982.

75. Wessells, R.J., Fitzgerald, E., Cypser, J.R., Tatar, M., and Bodmer, R. (2004). Insulin regulation of heart function in aging fruit flies. Nat. Genet. 36, 1275-1281.

76. Dietzl, G., Chen, D., Schnorrer, F., Su, K.C., Barinova, Y., Fellner, M., Gasser, B., Kinsey, K., Oppel, S., Scheiblauer, S., et al. (2007). A genome-wide transgenic RNAi library for conditional gene inactivation in Drosophila. Nature 448, 151-156.

77. Fink, M., Callol-Massot, C., Chu, A., Ruiz-Lozano, P., Izpisua Belmonte, J.C., Giles, W., Bodmer, R., and Ocorr, K. (2009). A new method for detection and quantification of heartbeat parameters in Drosophila, zebrafish, and embryonic mouse hearts. BioTechniques 46, 101-113.

78. Ocorr, K., Fink, M., Cammarato, A., Bernstein, S., and Bodmer, R. (2009). Semiautomated Optical Heartbeat Analysis of small hearts. Journal of visualized experiments : JoVE.

79. Yu, M.S., Spiering, S., and Colas, A.R. (2018). Generation of First Heart Field-like Cardiac Progenitors and Ventricular-like Cardiomyocytes from Human Pluripotent Stem Cells. J Vis Exp.

\section{FIGURE LEGENDS}

FIGURE 1: Knockdown (KD) of either subunit of Nascent polypeptide Associated Complex led to complete histolysis of the heart in adult flies. Hand4.2-GAL4 driving heart specific expression of A, control B, Nac $\alpha$-RNAi or C, bicaudal-RNAi. KD of Nac $\alpha$ or bicaudal led to an absent heart in adult flies. * indicates absence of the heart tube. Arrowheads point to remnants of alary muscles that normally attach to the heart for structural support (anterior-left). Pericardin, a heart-specific collagen is largely absent, except for remnants in the posterior end. D-F, Fluorescently tagged hearts using tdtK were imaged in vivo at white pupae stages which displayed structurally intact hearts in D, control, E, Nac $\alpha$-RNAi and F, bicaudal-RNAi expressing hearts, suggesting that the heart histolyzes during cardiac remodeling during metamorphosis. Arrow heads point to the internal valves that separate the larval aorta from the heart. ^ point to the inflow tracts called ostia.

FIGURE 2: Effect of Nac $\alpha$ knockdown (KD) using various GAL4 drivers on heart function and structure. A,B Structural and functional parameters measured by SOHA to assess the fly heart. Dotted lines indicate the heart tube borders. White solid vertical lines indicate the diameters of 
the heart, while horizontal lines indicate the duration of contraction/relaxation being measured. $\mathbf{A}$, Diastolic Diameter measures the heart diameter when it is fully relaxed, while systolic diameter measures the heart diameter when it is fully contracted. B, Motion-mode (m-mode) of the heart for temporal resolution of heart movement. Diastolic Interval measures the duration during which the heart is non-contractile, which occurs in this denervated fly preparation. Systolic Interval measures the duration that the heart is in active contraction and relaxation. $\mathbf{C}$, Both tinHE-GAL4 and tinC $44-G A L 4$ cardiac drivers reduced Diastolic Diameter when used to KD Nac $\alpha$ expression. Dot-GAL4 pericardial cell driver had no effect on diastolic diameter. D, Cardiac drivers had no significant effect on systolic diameters, while Dot-GAL4 increased systolic diameter slightly, indicating mild systolic dysfunction. E, Fractional Shortening is significantly decreased using both tinHE-GAL4 and tinCA4-GAL4 driver, while Dot-GAL4 had no effect. No changes in Heart Period F, or Diastolic Interval G, were detected with either cardiac drivers or Dot-GAL4. H, A slight reduction in systolic interval was detected when $N a c \alpha$-RNAi was driven with tinC 44 -GAL4. I, Adult fly hearts were stained with phalloidin to examine cytoskeletal structures following Nac $\alpha \mathrm{KD}$ using various tissue drivers. Compared to controls that display well- and tightly organized circumferential fibers that drive heart contractions, both tinHE-GAL4 and tinCA4-GAL4 drivers led to alterations in the organization of fibers. White arrowheads point to gaps in the fibers in KD samples consistent with the observed reductions in fractional shortening. KD of Nac $\alpha$ expression using Dot-GAL4 did not cause alterations in circumferential organization.

FIGURE 3: Nac $\alpha$ and the Hox gene Abdominal $B(A b d-B)$ genetically interact in the heart. A, The embryonic/larval fly heart remodels into adult structures through trans-differentiation of the Ubx and $a b d-A$ expressing cardiomyocytes into the adult heart and terminal chamber, respectively. The cardiomyocytes of the posterior larval heart that express $A b d-B$ (abdominal segment 6-7) histolyze and are absent in the adult heart. $\mathbf{B}$, Overexpression of $A b d-B$ in the heart using the cardiac driver Hand4.2-GAL4 led to complete absence of the adult heart, which in early stages of pupation was still present (see D). * indicates absence of the heart tube. C-E. Immunohistochemistry of pupal hearts 26-28 hours After Puparium Formation (APF). Dashed lines mark the region of the heart where orthogonal cross-sections were taken to examine Abd-B and DAPI expression in the nuclei. Images of the cross-section of cardiomyocytes and nuclei are displayed in the right panel. Arrowheads indicate either cardiac and pericardial nuclei that are both Abd-B and DAPI positive. C, In controls, phalloidin stained the circumferential fibers of the pupal aorta and heart. Abd-B staining was not detected in the cardiomyocyte nuclei within aorta and anterior heart segments but, ABDB is stained in the posterior segments of the embryo. D, 
Overexpression of $A b d-B$ using Hand4.2-GAL4 resulted in strong Abd-B staining in the nuclei (as indicated by the arrowheads) throughout the pupal heart and pericardial cells prior to remodeling. Cross section clearly shows that Abd-B is localized in the nucleus. E, Knockdown of Nac $\alpha$ in the heart resulted in ectopic Abd-B expression throughout the heart tube. Staining was present within the nuclei (marked by DAPI), cytoplasm and heart lumen (see orthogonal sections, right). F-H. Concurrent knockdown of Nac $\alpha$ and $A b d-B$ in the heart, $\mathbf{G}$, led to rescue of heart tube formation, with visible circumferential fibers (arrowheads) albeit less well-organized than controls. Rescued hearts contracted as visualized in the M-mode (right panel). $\mathbf{H}$, Rescued hearts were smaller in diastolic diameter with no change in systolic diameter resulting in reduced Fractional Shortening (FS). This effect was not due to titration of GAL4 onto 2 UAS sites, as combination of UAS-Nac $\alpha$ RNAi with UAS-Val10-GFP still produced a no heart phenotype. I, Arrow indicates remnants of the ventral longitudinal muscle in the anterior end of the abdomen.

FIGURE 4: Temporal regulation of $N a c \alpha$-RNAi expression in the heart. Using a temperature inducible driver specifically in the heart (HTT, Hand4.2-GAL4, tubulin-GAL80 ${ }^{\text {ts; }}$ tubulin-GAL80 ${ }^{\text {ts }}$ ), Nac $\alpha$-RNAi was expressed during specific stages of development by controlling ambient temperature to determine its contribution to cardiogenesis. Controls lacking RNAi are kept in similar temperature conditions to account for any developmental effects of temperature on the heart. A-H. Phalloidin staining to visualize cytoskeletal structural effects of Nac $\alpha$ knockdown (KD). A, As a test of GAL80 control of transcription, flies held at $18^{\circ} \mathrm{C}$ throughout development did not produce changes to heart structure indicating an inhibition of Nac $\alpha$-RNAi transcription. B, Exposing flies to high temperatures $\left(28^{\circ} \mathrm{C}\right)$ throughout development produced a no heart phenotype similar to the effects of driving Nac $\alpha$-RNAi using Hand4.2-GAL4 alone, suggesting an induction of Nac $\alpha$-RNAi transcription and subsequent Nac $\alpha$ KD with exposure to higher temperatures. * indicates absence of the heart. C, Exposing flies to high temperature during adulthood only for 1 week, D, pupae to eclosion, or E, mid-larvae to eclosion did not produce gross structural defects in the heart. F, Exposing embryos to high temperatures starting at egglay up until 24 hours resulted in the absence of the terminal chamber, indicated by white bar. Only thin alary muscles were present. G, Extending the high temperature exposure to 48 hours led to similar loss of the posterior heart, indicated by white bar. $\mathbf{H}$, Only when the hearts were exposed to higher temperatures during embryonic stage (24 hours) and pupal stage until eclosion, were we able to recapitulate the no heart phenotype produced by exposing the heart to constant high temperatures. I, Functional analysis of the adult heart following Nac $\alpha$ KD at various 
developmental stages. Maintaining flies at $18^{\circ} \mathrm{C}$ throughout development or exposure of adult flies to high temperature for 1 week led to no changes in diastolic diameter, systolic diameter, or fractional shortening. High temperature exposure from pupae to eclosion or from mid-larvae to eclosion led to subtle changes in diameters and fractional shortening. Exposure of embryos to high temperatures for $24 \mathrm{hr}$ led to constricted diastolic and systolic diameters. ${ }^{*} p<0.05,{ }^{* *} p<0.01$, ${ }^{* * *} p<0.001$.

FIGURE 5: Nac $\alpha$ and Hox genes interact to redirect differentiation of Multipotent Cardiac Progenitors (MCPs). A,C,E, Quantitation of differentiated cell populations 9 days after siRNA treatment. B,D,F. Representative images of immunohistological staining for select conditions. A,B, Total Cell Populations following siRNA treatment were not significantly changed compared to controls, except for Gata4/6,MyoCD siRNA condition which reduced overall cell count. Knockdown (KD) of Naca (green), HOXC12 (light blue), HOXD12 (light gray) singly resulted in cell populations that trended lower. This decrease was reversed and significantly different upon combined transfection of Nac $\alpha$ siRNAs with Hox genes, compared to single siRNA transfections. C,D, Nac $\alpha$ KD alone significantly decreased the proportion of cardiomyocytes (ACTN1+) compared to controls, while treatment with HOXC12 or HOXD12 siRNA individually, had no effect. Combined transfection of Nac $\alpha$ and Hox genes reversed the decrease in cardiomyocyte population and was significantly different compared to Nac $\alpha$ KD alone and no longer different compared to controls. Gata4/6,MyoCD KD also significantly lowered the proportion of cardiomyocyte populations. E,F, Nac $\alpha$ KD increased the proportion of fibroblasts (TAGLN+) compared to controls. Combined KD of Nac $\alpha$ with any of the Hox genes did not alter fibroblasts numbers compared to controls but were significantly reduced compared to Nac $\alpha$ KD alone. G, Images of merged staining of cardiomyocyte, fibroblast, and endothelial cells. Significance * vs. control. ^ vs. Nac $\alpha$. \# comparison is indicated by line. ${ }^{*} p<0.05,{ }^{* *} p<0.01,{ }^{* * *} p<0.001,{ }^{* * * *}$ $p<0.0001$.

FIGURE 6: Knockdown (KD) of SRP subunits in the Drosophila heart caused distinct heart defects. A, The Signal Recognition Particle (SRP) is composed of an RNA molecule holding together 6 SRP subunits. B-M, Individual SRP subunits were KD by RNAi using the heart specific driver Hand4.2-GAL4 and adult flies were stained with phalloidin to determine their contribution to heart structure and development. * indicates missing heart segments or cardiomyocytes. KD 
of C, SRP9 and D, SRP14 subunits, responsible for elongation arrest during translation, did not lead to gross alterations in heart structure and were comparable to B, controls. E, KD of SRP68 led to complete loss of the heart. G, Interestingly, KD of SRP72, a binding partner to SRP68 led to the presence of a heart tube. $\mathbf{H}$, Higher magnification of the conical chamber, marked by an arrowhead, showed that the conical chamber was constricted compared to controls, and resembled a larval aorta. F, KD of SRP19, led to a partial heart phenotype, where the anterior region of the heart was absent but the posterior end remained. I, KD of SRP54 led to a heart tube but with missing heart cells (indicated by asterisks) in random regions of the heart. $\mathbf{J}$, Higher magnification of missing cardiomyocyte. K, KD of the SRP Receptor- $\beta$, a subunit of the receptor anchored to the ER membrane that binds to the SRP-ribosome-nascent chain complex, led to segments of the heart, usually the valves, that were constricted and larval like, indicated by the arrowhead. L, Higher magnification of the narrowed heart tube. Ostia structures are still present as marked by ${ }^{\wedge}$. M, As comparison, control valve cells in controls are wider than SRP $\beta$ KD and are densely packed with myofibrils.

Figure 7: A schematic diagram of protein translation at the ribosome exit site, where nascent polypeptide cofactors NAC and SRP select for gene targets, regulating their expression in a tissue and/or cell specific manner to regulate heart morphogenesis. Our work suggests that the posterior determining Hox gene $A b d-B$ is a target of $N a c \alpha$, as the knockdown of $N a c \alpha$ led to $A b d-B$ misexpression and disruption of heart morphogenesis. Knockdown of SRP subunits each led to distinct heart defects: SRP72 knockdown disrupts conical chamber morphogenesis, SRP-R $\beta$ targets internal valve cells, SRP54 leads to missing cardiomyocytes, SRP19 leads to loss of anterior segment of the heart, while SRP68 led to a no heart phenotype similar to Nac $\alpha$ knockdown. Targets of SRP have yet to be determined.

\section{SUPPLEMENTAL FIGURE LEGENDS}

SUPPLEMENTAL FIGURE 1: A, Still images of remodeling pupal hearts with control, Nac $\alpha$ knockdown (KD) and bic KD. At 24hr APF, the early pupal heart is present in controls (left) and Nac $\alpha$ (middle) and bic (right) knockdown flies. The internal valves (yellow arrowheads) are visible which separate the larval aorta (left) and the heart (right) which we use as a landmark through remodeling. At about 34hr APF, the internal valves are present but the aorta is more difficult to visualize as the heart transdifferentiates. At approximately 60hr APF, the remodeling adult heart 
tube is visible in controls with identifiable ostia structures (marked by ${ }^{\wedge}$ ). In Nac $\alpha$ and bic $\mathrm{KD}$ hearts, no heart tube is visible and the area is filled in by rounded fat cells (green arrowheads). The area of the embryo with fluorescent signal (circled) are remnants of histolyzed cardiomyocytes that slowly disperse and weaken in intensity. B, Pupal dissections at approximately 24-26hr APF stained with phalloidin shows the presence of the fly aorta and heart in controls (left) and with Nac $\alpha \mathrm{KD}$ (right) at two magnifications. Arrows point to the presence of a heart tube.

SUPPLEMENTAL FIGURE 2: Knockdown of $A b d-B$ using heart specific driver Hand4.2-GAL4 led to intact hearts with posterior ends (indicated by arrowhead) that were more prominent and dilated compared to controls suggesting incomplete histolysis during cardiac remodeling.

SUPPLEMENTAL FIGURE 3: Overexpression of an inhibitor of apoptosis (Diap1) concurrently with $N a c \alpha-R N A i$ does not rescue the loss of the heart. * indicates absence of the adult heart tube.

SUPPLEMENTAL FIGURE 4: Testing interaction of Nac $\alpha$ and Hox gene $A b d-B$ using the weaker cardiac specific tinHE-GAL4 driver by $\mathbf{A}$, functional, B, temporal and $\mathbf{C}$ structural assessment. A, Knockdown (KD) of Nac $\alpha$ (combined with UAS-Stinger::GFP to control for UAS binding sites) using tinHE-GAL4 caused a decrease in both diastolic and systolic diameters that produced a slight but not significant decrease in fractional shortening. KD of $A b d-B$ (combined with UASStinger::GFP) did not produce significant changes in fractional shortening or diameters compared to control but fractional shortening and diastolic diameters were significantly higher compared to Nac $\alpha$; Stinger genotype. Combined knockdown of Nac $\alpha$ and $A b d-B$ produced heart parameters that were not different to controls but recapitulated heart function produced by $A b d-B \mathrm{KD}$ alone, suggesting that the heart function was rescued. B, Temporal parameters were unchanged with $N a c \alpha-R N A i$ expression. KD of $A b d-B$ lengthened systolic interval compared to controls. Combined Nac $\alpha$ and $A b d-B$ KD displayed longer systolic intervals similar to $A b d-B$ KD alone, suggesting a rescue. C, Phalloidin staining of select genotypes. Compared to controls, Nac $\alpha$ knockdown disrupted circumferential fiber organization creating gaps in the matrix (similar to Figure 2I). KD of $A b d-B$ did not significantly alter circumferential fiber organization. Combined knockdown of $N a c \alpha$ and $A b d-B$ (2 examples shown) improved circumferential fiber organization compared to Nac $\alpha$ knockdown alone. * vs control KKGD. ^ compared to Nac $\alpha$,Stinger. * $p<0.05$, ${ }^{* *} p<0.01,{ }^{* * *} p<0.001$. 
SUPPLEMENTAL FIGURE 5: Nac $\alpha$ and Hox genes interact to redirect differentiation of Multipotent Cardiac Progenitors (MCPs). A, Knockdown (KD) of Nac $\alpha$, Hox genes or their combination did not produce a significant change in the proportion of endothelial cell (CDH5+). The KD of transcription factors Gata4/6,MyoCD increased the proportion of endothelial cells. B, Representative images of immunohistological staining for select conditions.

SUPPLEMENTAL FIGURE 6: Images of fluorescently labeled (tdtK) larval aorta and heart (abdominal segments 3 and 4 ) of White Pupae, which showed that heart tubes were present prior to cardiac remodeling with any of the SRP subunit knocked down using Hand4.2-GAL4. Internal valves separating the larval aorta and heart are marked by arrowheads.

SUPPLEMENTAL VIDEO 1, 2 and 3: Video of fluorescently labeled pupal hearts (tdtK) of control (Video 1), Nac $\alpha$ (Video 2) and bicaudal (Video 3) knockdown flies. Images were captured starting early pupae (about 6-7 APF) through cardiac remodeling up until about $80 \mathrm{hr}$ APF.

SUPPLEMENTAL TABLE 1: Table of transgenic lines used in the study. 


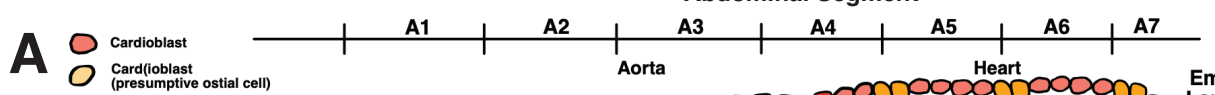

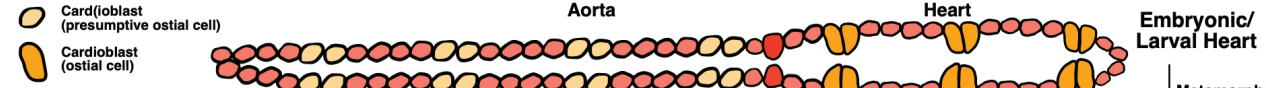

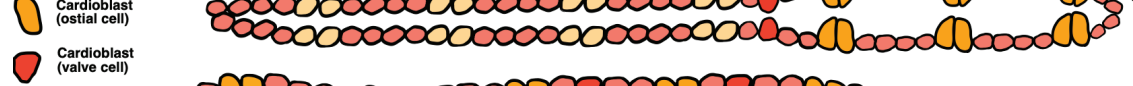

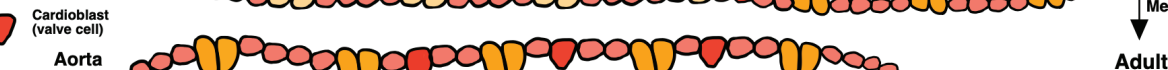

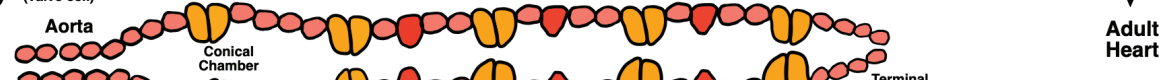

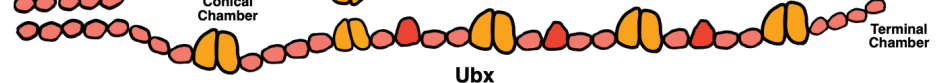

$$
\text { Ubx }
$$

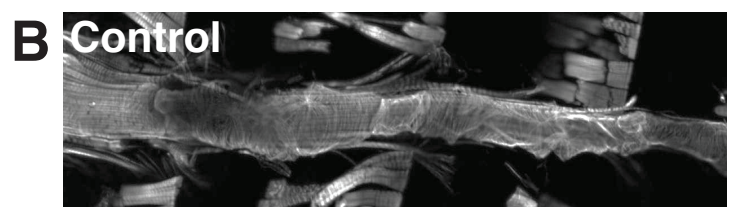

C Control
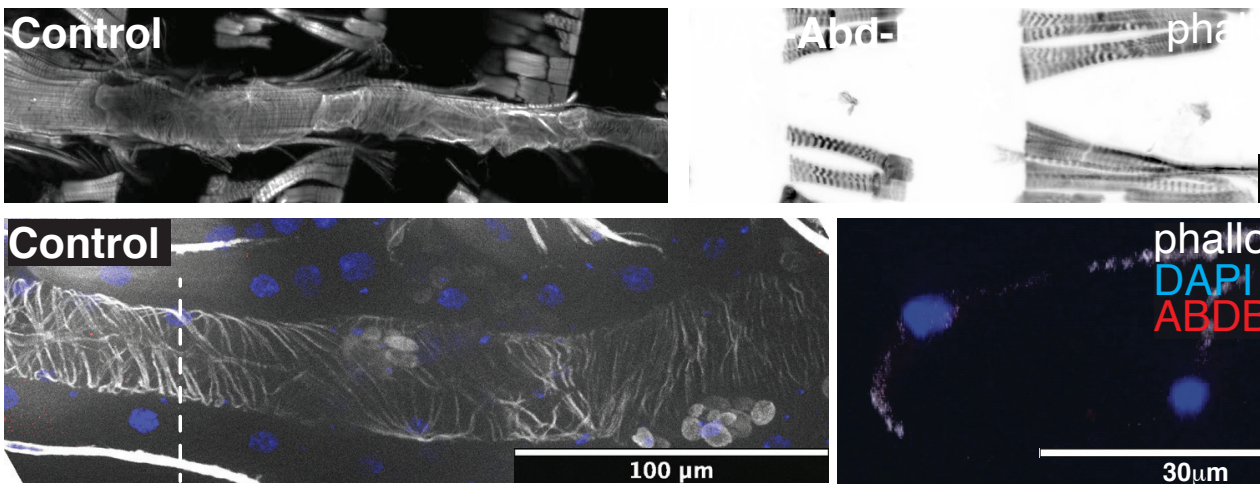

Metamorphosis

100 um
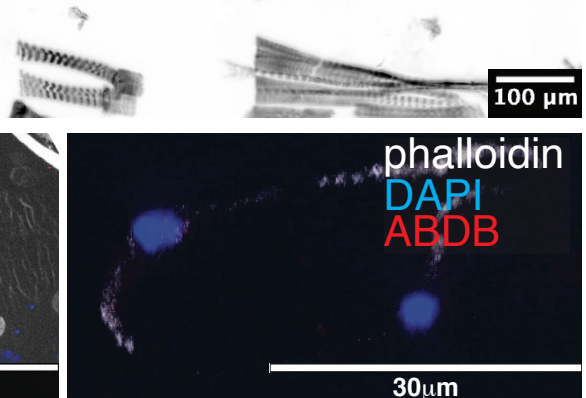

\section{UAS-Abd-B}
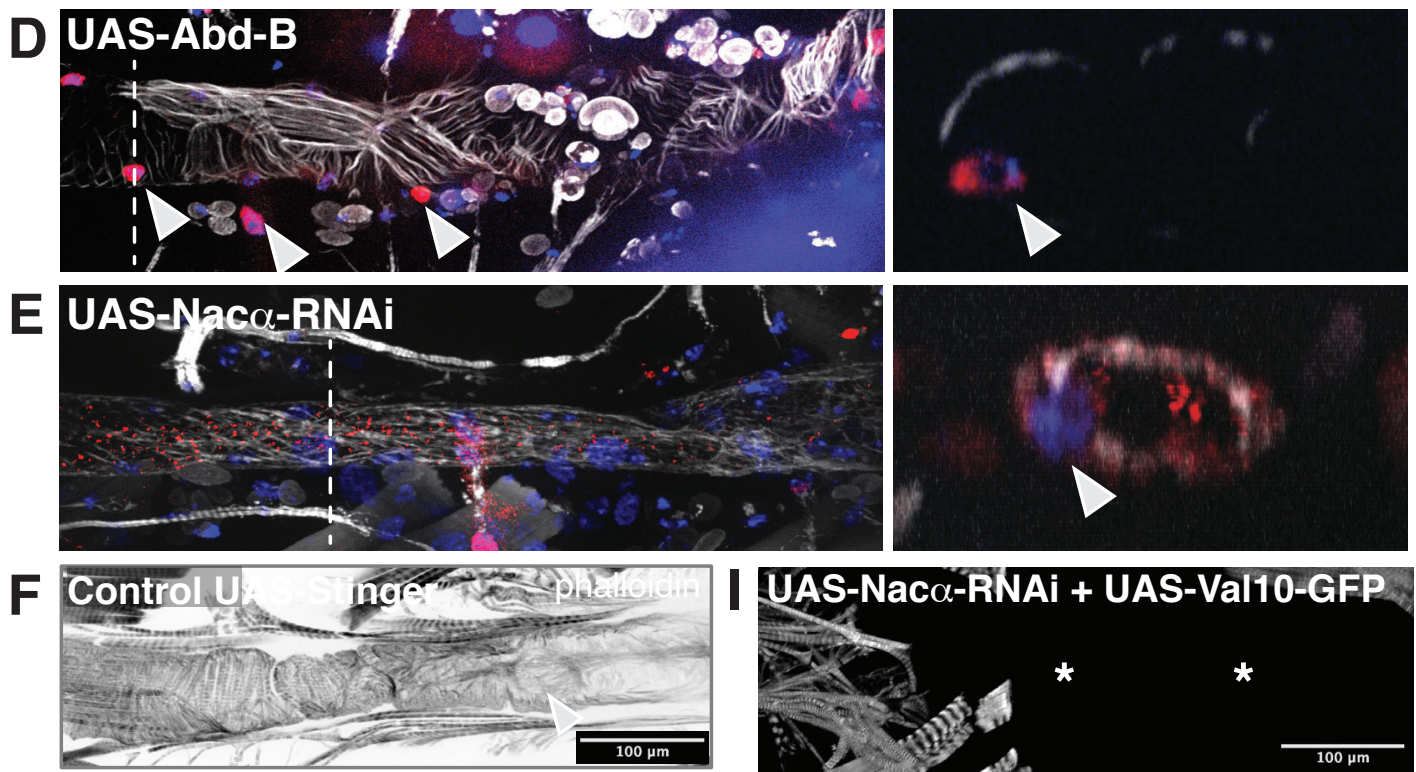

G
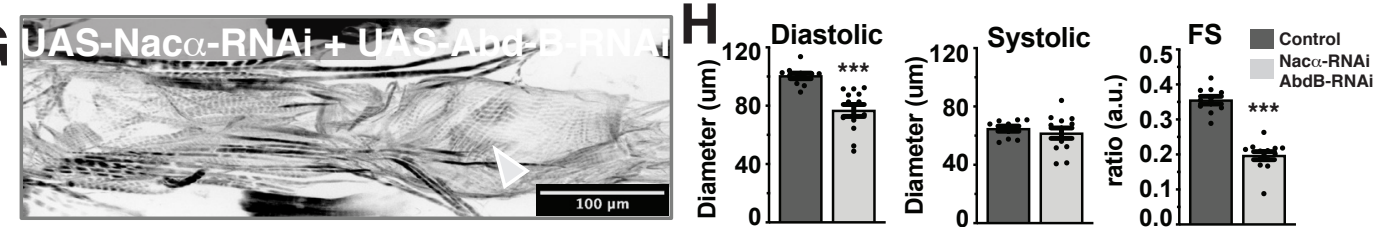
Nac $\alpha-R N A i$

A None

B Throughout

Development

C Adult

D Pupae-eclosion

E Mid-larvae-eclosion
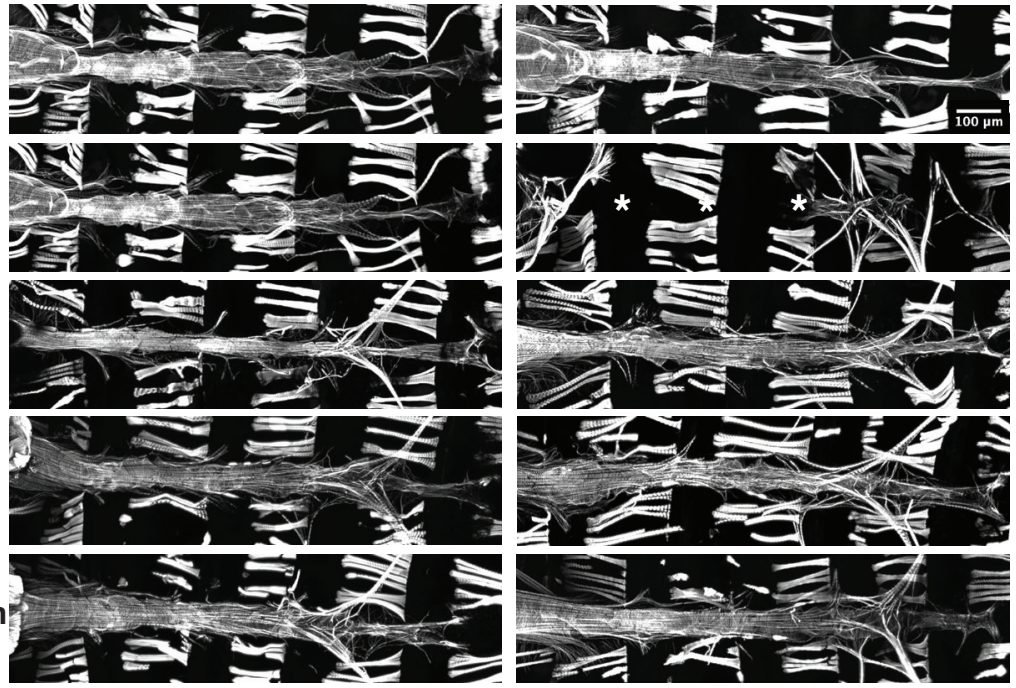

F Embryo 24 hours

G Embryo 48 hours

Hembryo + pupae
only
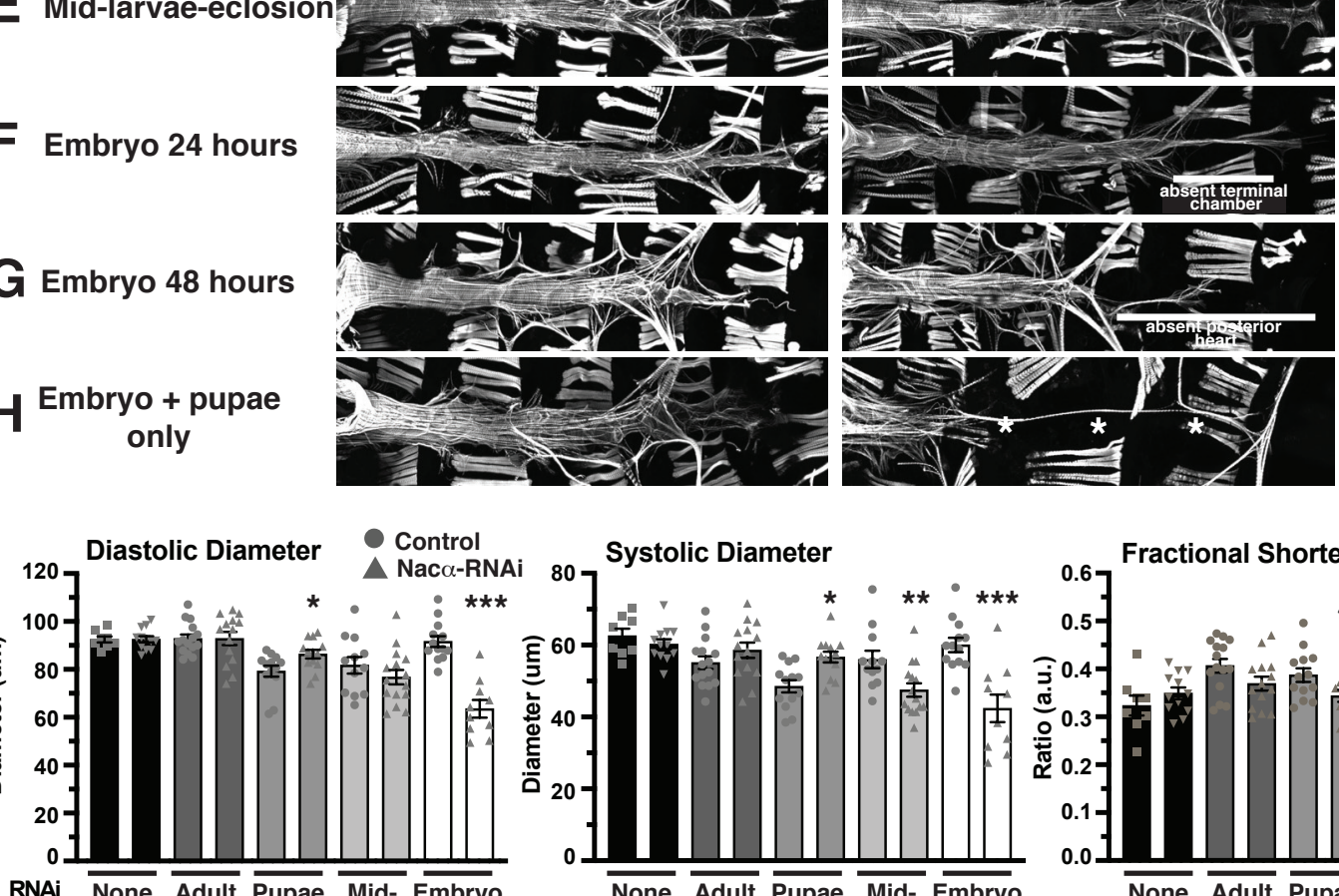
Induction
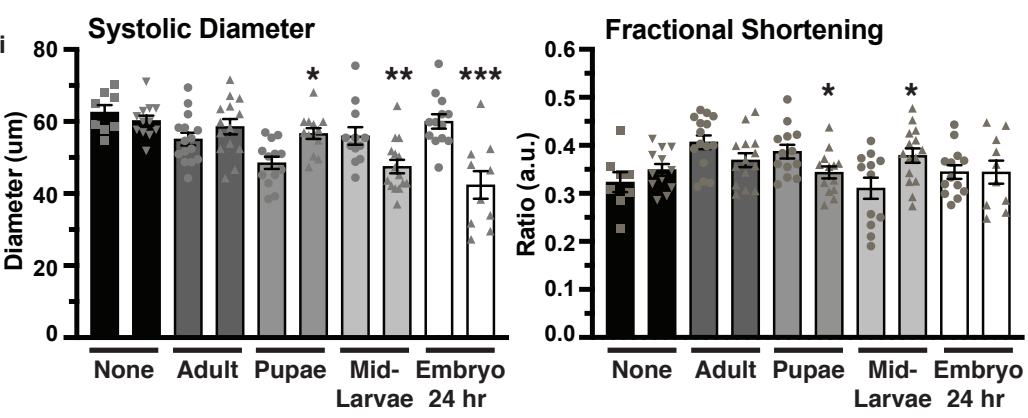
FIGURE 5
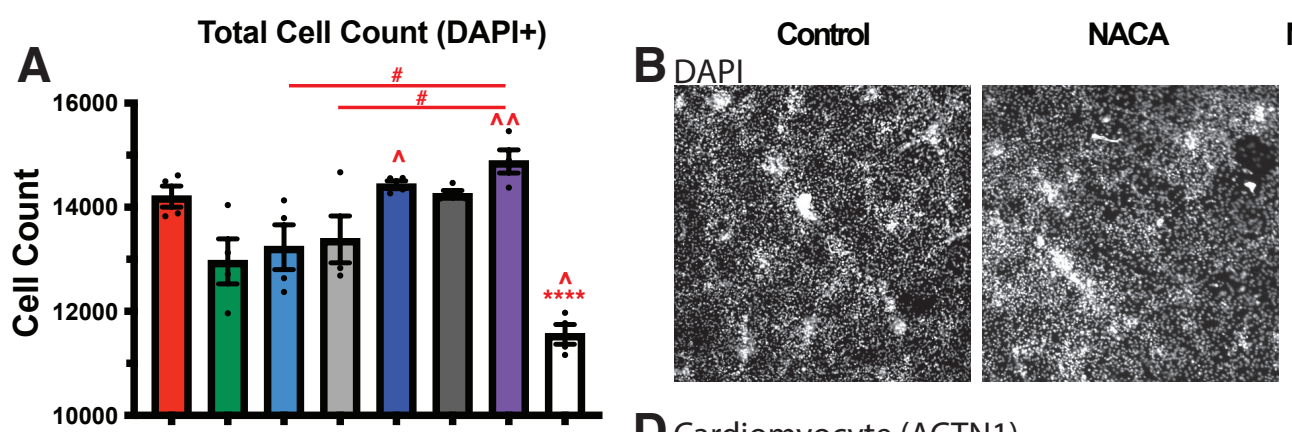

NACA HOXC12 HOXD12

GATA4/6 MYOCD

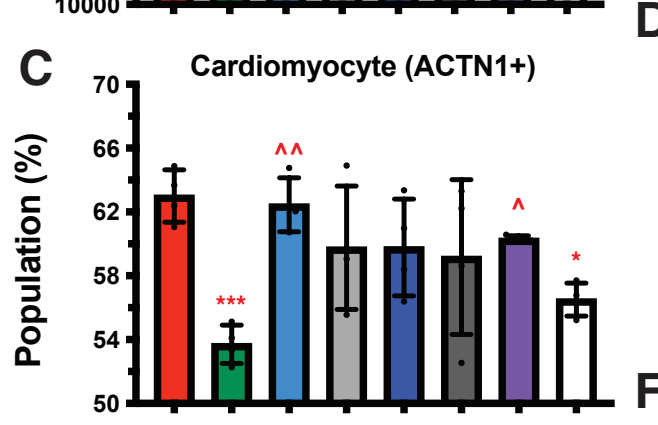

Cardiomyocyte (ACTN1)
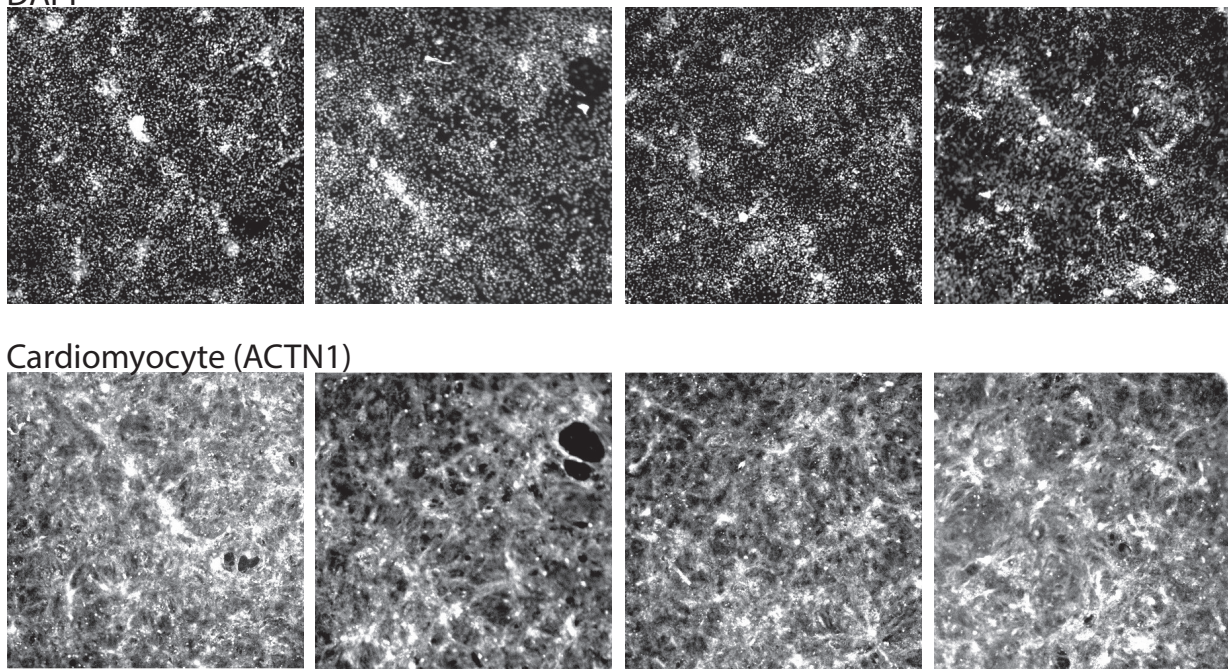

F Fibroblast (TAGLN+)
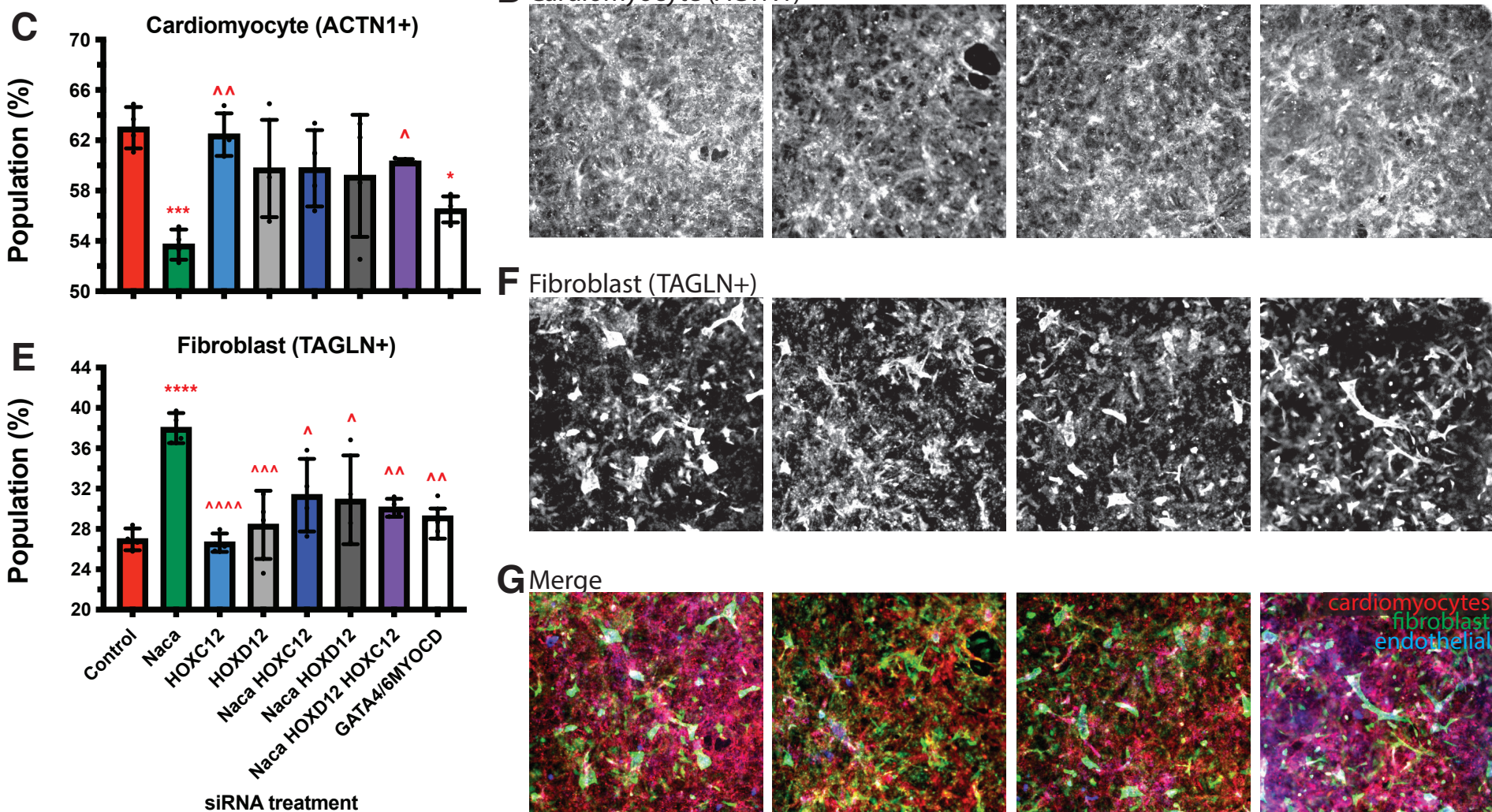

GMerge
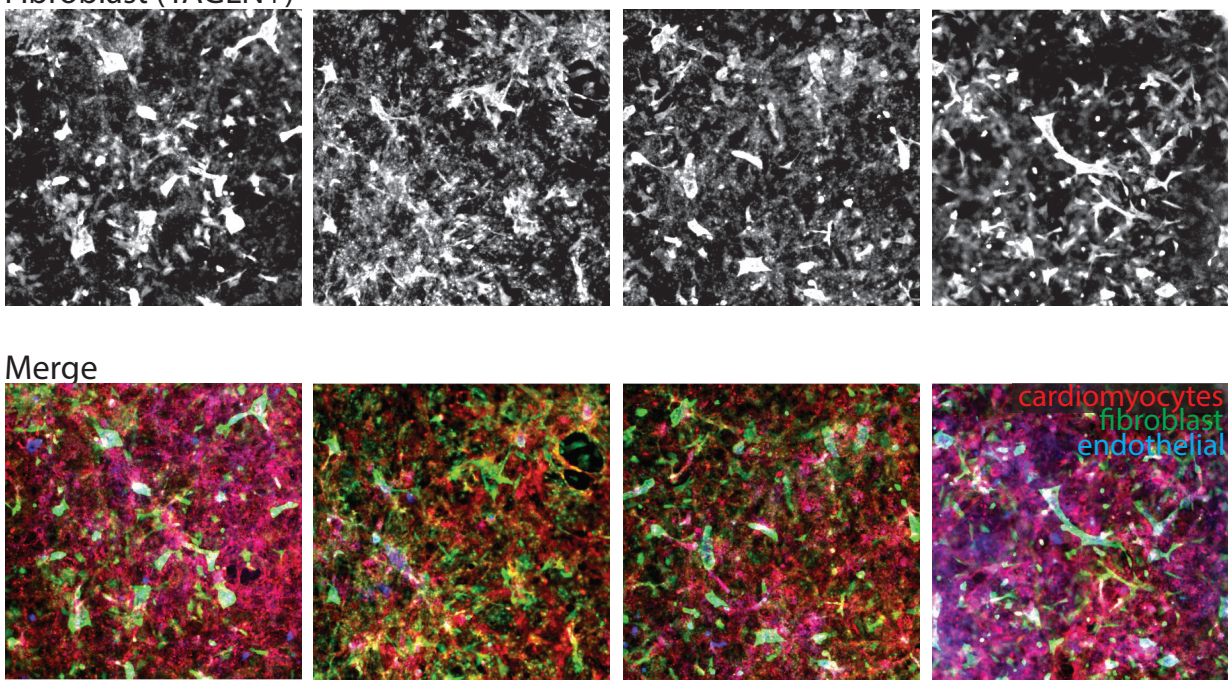

siRNA treatment
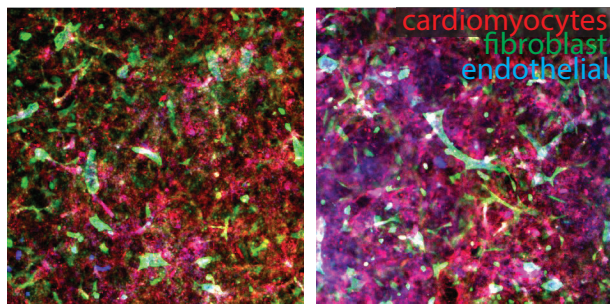


\section{FIGURE 6}

A

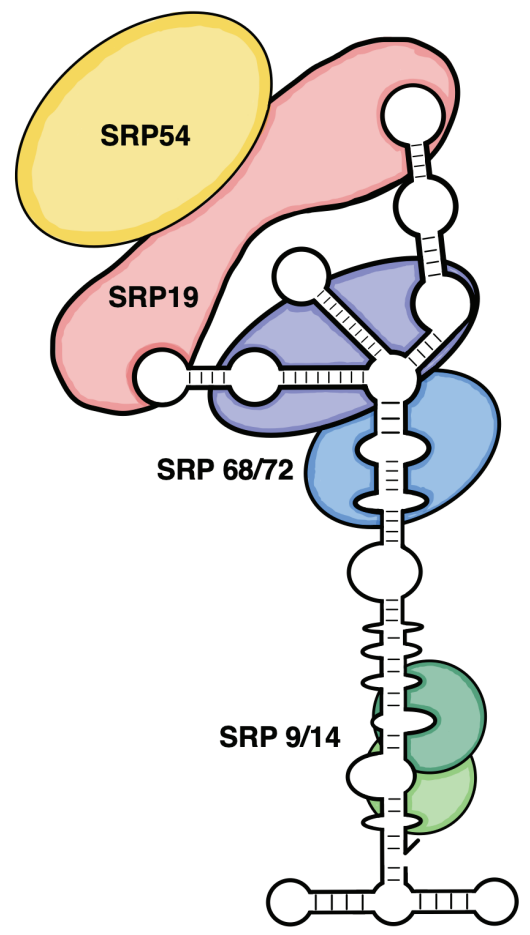

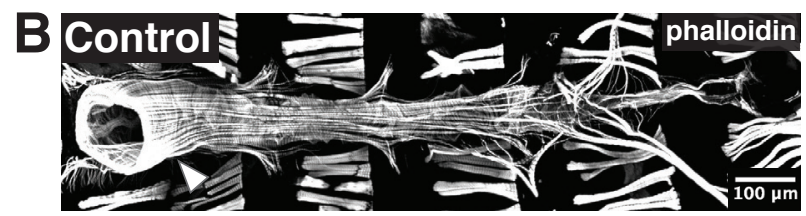

C SRP9-RNAI

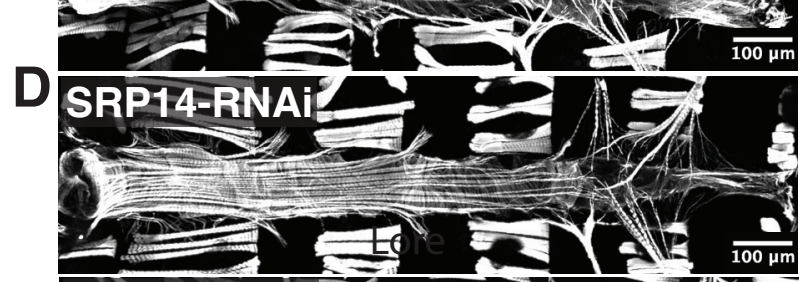

E SRR68-RNAA

$F$ SRPI9RNAT

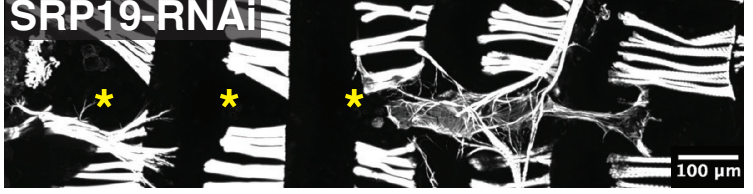

G SRP72-RNAD,

I

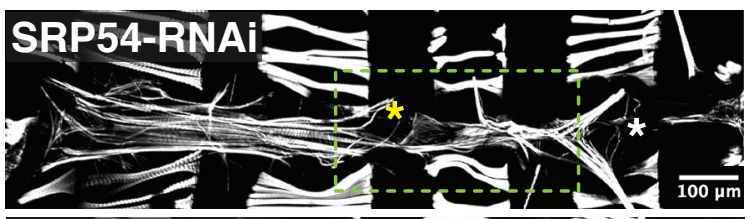

$K$ SRPAB-RNAI:-
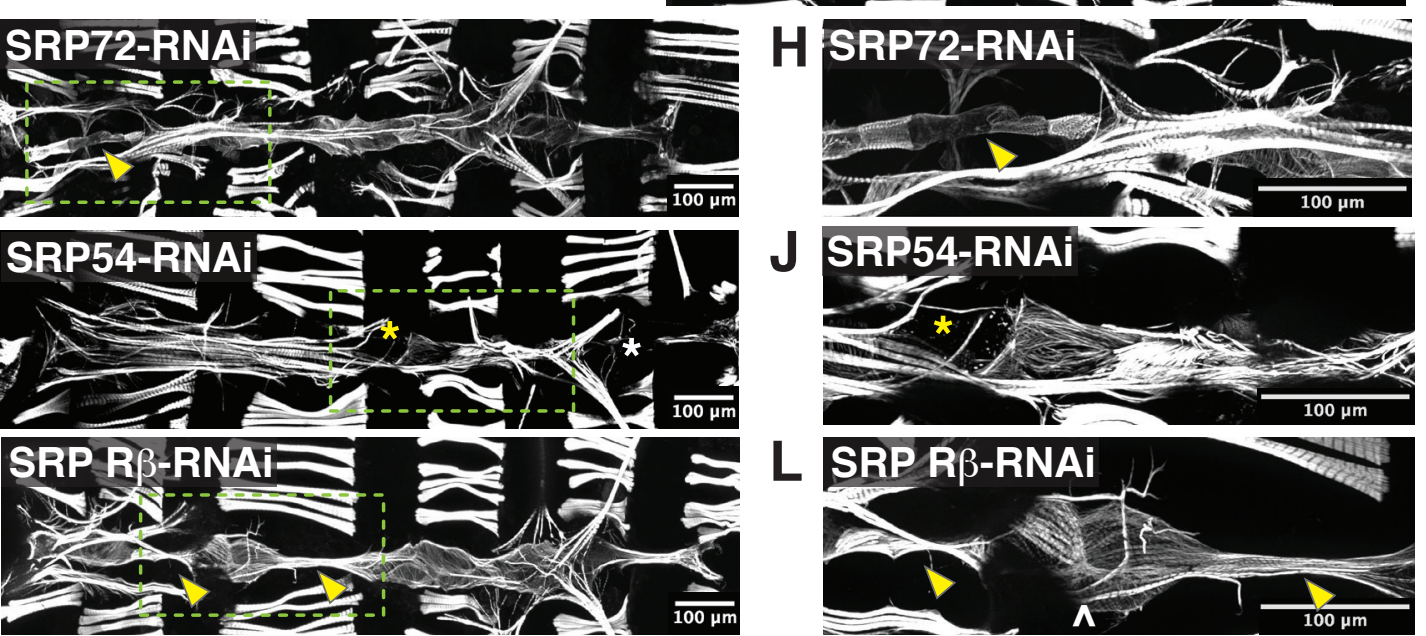

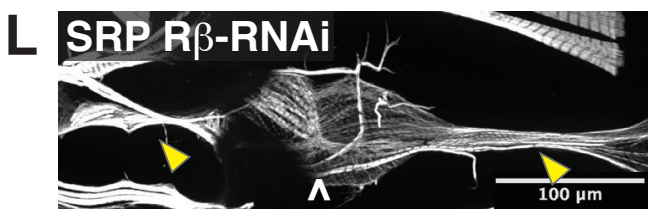

MControl + a wasere

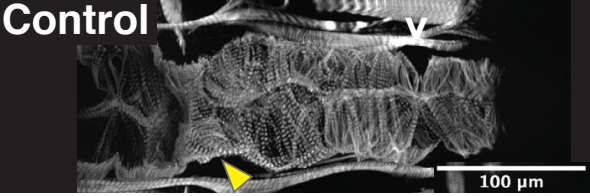


FIGURE 7

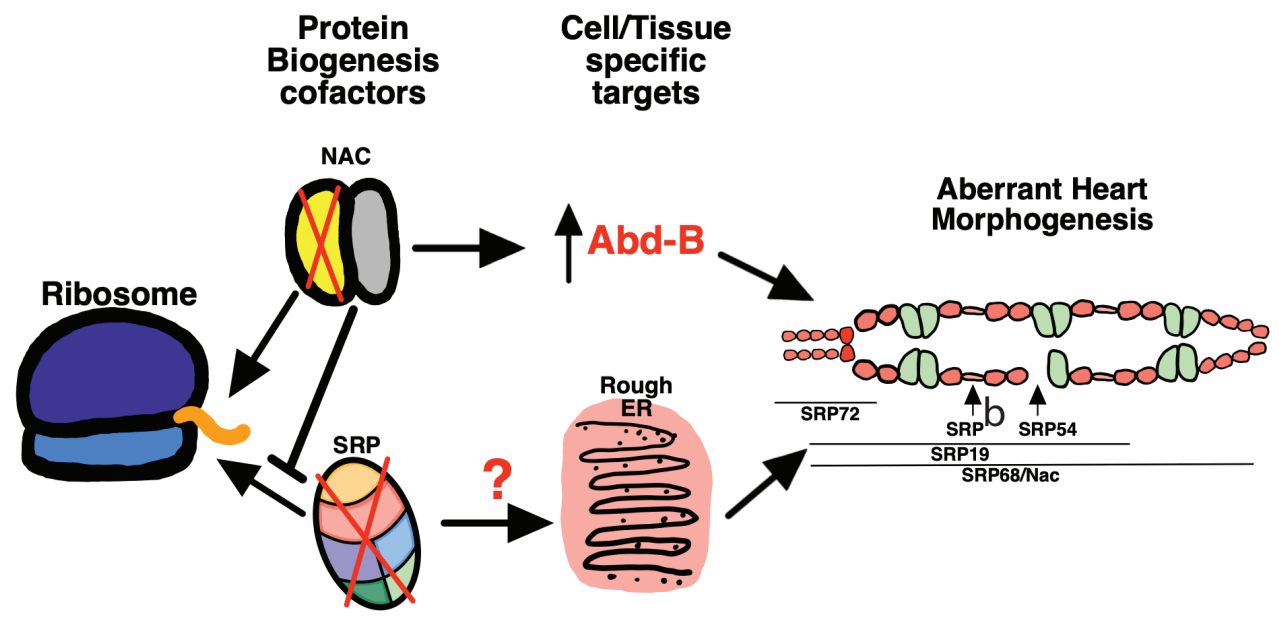




\section{Supplemental Figure 1}

A Hand4.2-GAL4, td-tomato $x$

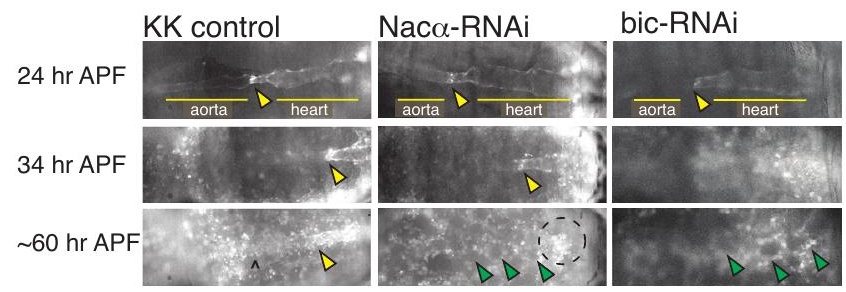

B

28hr After Puparium Formation

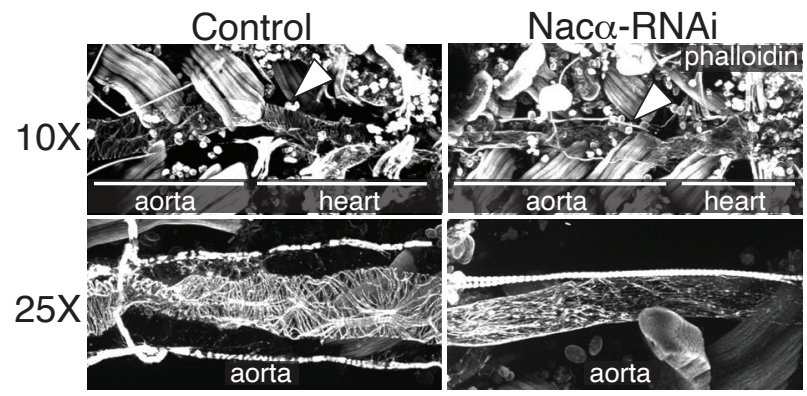

\section{Supplemental Figure 2}
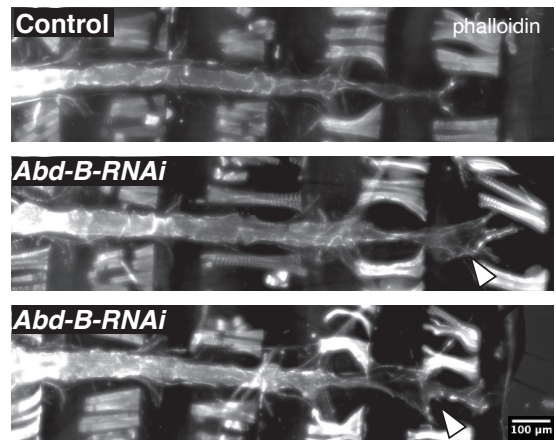


\section{Supplemental Figure 3}

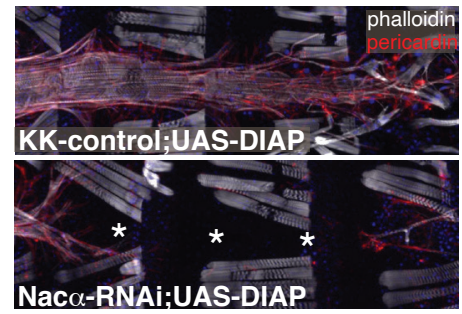

\section{Supplemental Figure 4}

Rescue using TinHEGAL4

A
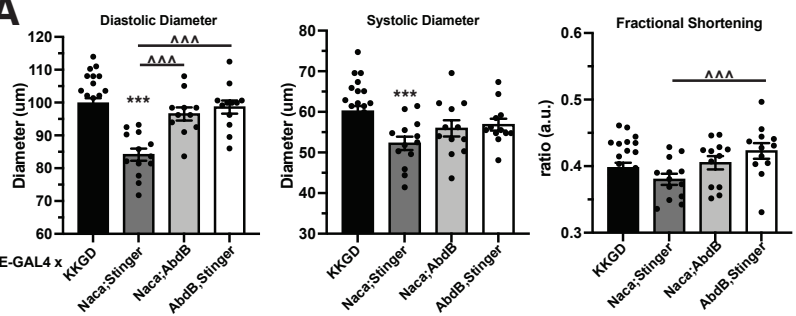

B
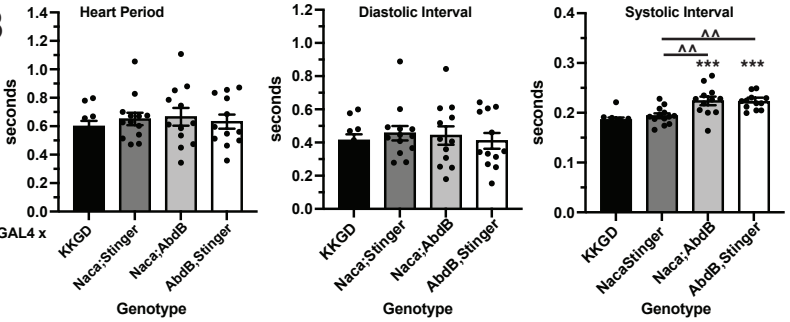

C TinHE-GAL4 $x$
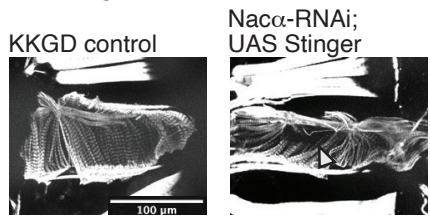

AbdB-RNAi;

UAS Stinger

Nac $\alpha-R N A i$;

AbdB-RNAi

Naco-RNAi;
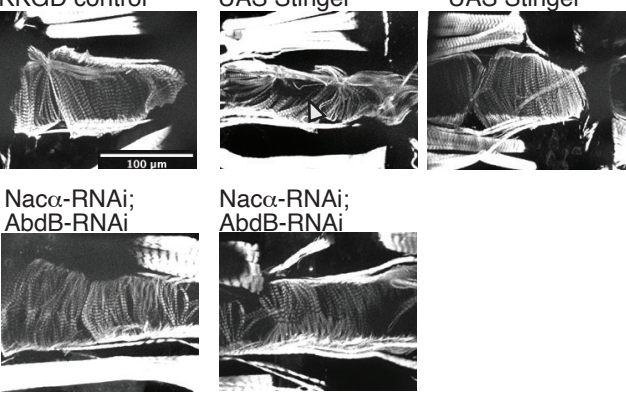


\section{Supplemental Figure 5}

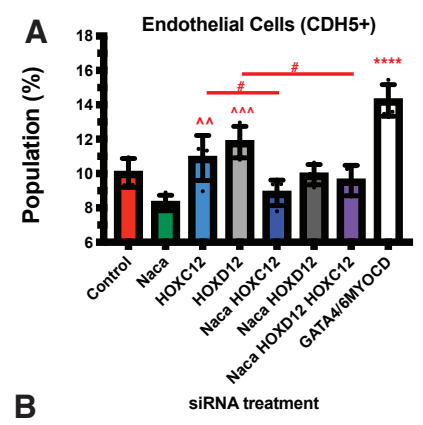

Endothelial (CDH5)

Control

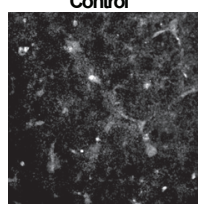

NACA

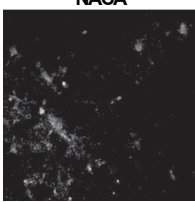

NACA HOXC12 HOXD12

GATA4/6 MYOCD

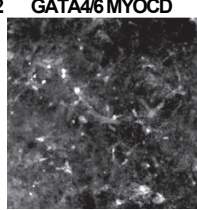




\section{Supplemental Figure 6}
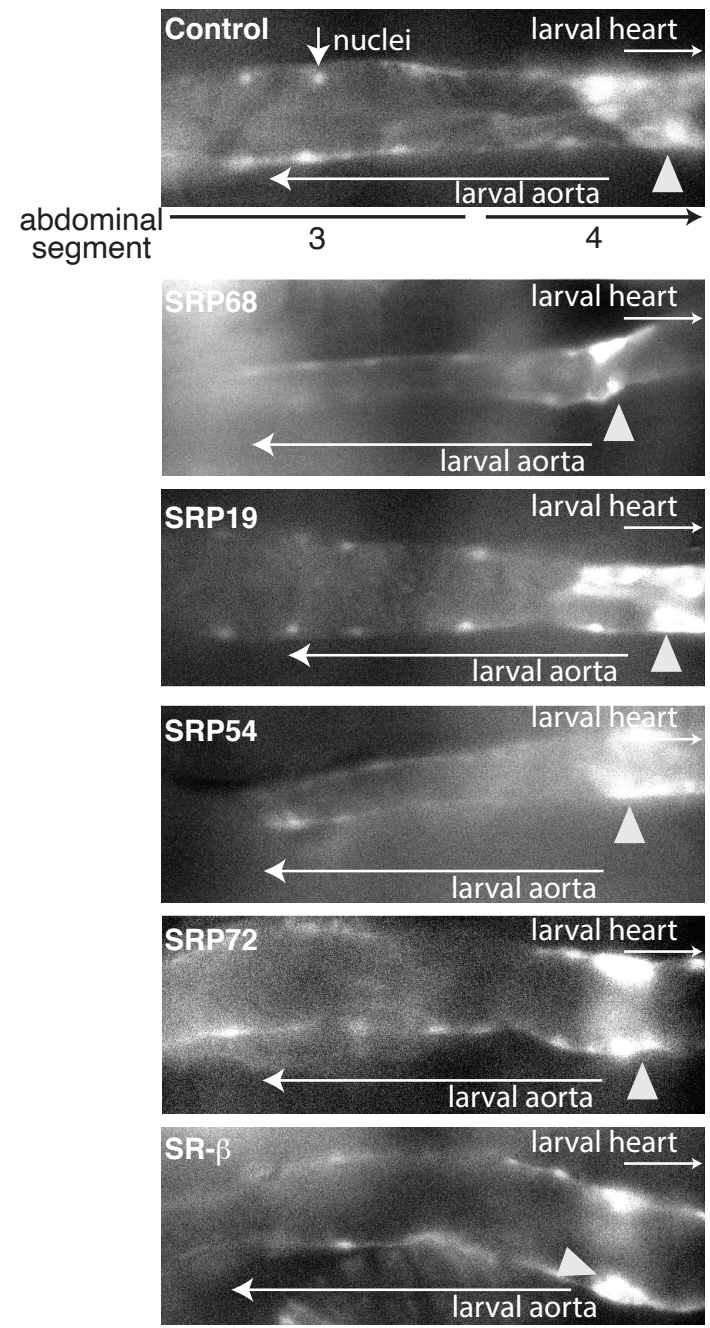
bioRxiv preprint doi: https://doi.org/10.1101/2022 0124.477419. this version posted January 24,2022. The copyright holder for this preprint (which was not certified by peer review) is the author/funder, who has granted bioRxiv a license to display the preprint in perpetuity. It is made available under aCC-BY-NC-ND 4.0 International license.

SUPPLEMENTAL TABLE 1

\begin{tabular}{|c|c|c|c|c|}
\hline Fly gene & Human Gene & $\begin{array}{l}\text { DIOPT } \\
\text { score }\end{array}$ & $\begin{array}{c}\text { Fly } \\
\text { Collection }\end{array}$ & ID/Stock \\
\hline Nac-alpha & $N A C A$ & 11 & VDRC & $\begin{array}{c}\mathrm{KK} v 109114 \\
\mathrm{GD} \text { v36017 }\end{array}$ \\
\hline bicaudal & $B T F 3 / N A C \beta$ & 12 & VDRC & $\begin{array}{c}\text { KK v104718 } \\
\text { GD v15453 }\end{array}$ \\
\hline$A b d B$ & $\begin{array}{l}\text { HOXC12 } \\
\text { HOXD12 } \\
\text { (multiple orth }\end{array}$ & $\begin{array}{c}3 \\
3 \\
\text { ologs) }\end{array}$ & VDRC & $\begin{array}{c}\text { KK v104872 } \\
\text { GD v12024 }\end{array}$ \\
\hline$U A S-A b d B$ & & & BDSC & 913 \\
\hline SRP9 & SRP9 & 13 & VDRC & $\begin{array}{c}\text { KK v105682 } \\
\text { GD v23678 }\end{array}$ \\
\hline SRP14 & SRP14 & 11 & VDRC & $\begin{array}{c}\text { KK v101444 } \\
\text { GD v23422 }\end{array}$ \\
\hline SRP19 & SRP19 & 14 & VDRC & KK v106756 \\
\hline SRP68 & SRP68 & 14 & VDRC & $\begin{array}{l}\text { KK v104867 } \\
\text { GD v273351 }\end{array}$ \\
\hline SRP72 & SRP72 & $\overline{14}$ & VDRC & $\begin{array}{l}\text { GD v43978 } \\
\text { GD v21641 }\end{array}$ \\
\hline SRP54 & SRP54 & 11 & VDRC & GD v51088 \\
\hline UAS-stinger & nuclear GFP & & BDSC & 84277 \\
\hline UAS-Val10-GFP & & & BDSC & 35786 \\
\hline tubulin-GAL80ts & Tubulin & & $\begin{array}{l}\text { BDSC } \\
\text { BDSC }\end{array}$ & $\begin{array}{l}7017 \\
7019\end{array}$ \\
\hline $\begin{array}{c}\text { Hand4.2-GAL4 } \\
\text { Hand4.2-GAL4; tdtK }\end{array}$ & Hand & & & \\
\hline tinHE-GAL4 & tinman & & & \\
\hline $\operatorname{tin} C D 4-G A L 4$ & tinman & & & \\
\hline Dot-GAL4 & $\begin{array}{c}\text { UDP } \\
\text { glucuronosyltransf } \\
\text { erase family }\end{array}$ & & & \\
\hline
\end{tabular}

